

\section{ABSTRACT}

We report on the design of a tangible device for encouraging the acceptance of unproductive time. We first conducted interviews for a better understanding of the subjective experience of productivity. We found that while the idea of being productive can evoke positive feelings of satisfaction, dealing with unproductive time can be a struggle, negatively affecting people's moods and self-esteem. These findings guided the design and implementation of RU, a tangible device for reflecting on self-care time. Our prototype offers a physical representation of the mainstream productivity mindset and plays with the idea of connecting and charging energy to encourage the user to experience the time considered unproductive as self-care. In a second study, participants used the device for 5 days and our results suggest that the device motivates reflection on activities beyond work and increases awareness of the importance of taking time for self-care. 


\section{SAMMANFATTNING}

I rapporten redogör vi utformningen av ett fysiskt verktyg vars syfte är att öka acceptansen för icke-produktiv tid. Först användes intervjuer för att skapa en bättre förståelse och insikt i vad en "produktiv upplevelse" är. Intervjuerna visade att, samtidigt som idéen av att vara produktiv kan ge positiva känslor i form av "uppfyllnad", så kan hanteringen av icke-produktiv tid vara jobbig och därmed negativt påverka människors humör och självkänsla. Insikterna från intervjuerna användes som stöd för designen och implementationen av RU, ett fysiskt verktyg vars användning är menad att härleda till reflektion samt tid för självvård. Prototypen är en fysisk representation av vad som anses var den stereotypiska bilden av ett produktivt sinne. Prototypen spelar på idéen av att koppla samman och ge energi i syfte om att motivera användaren att uppleva oproduktiv tid som självvård. I en ytterligare exekverad studie använde deltagarna RU under 5 dagar där resultatet indikerade på att verktyget motiverar till reflektion i aktiviteter bortom jobb och en ökad medvetenhet om vikten i att ta sig tiden för självvård. 


\title{
Designing A Tangible Device for Re-Framing Unproductivity
}

\author{
Judith Sirera i Pulido \\ KTH Royal Institute of Technology \\ Stockholm, Sweden \\ jsip@kth.se
}

\begin{abstract}
We report on the design of a tangible device for encouraging the acceptance of unproductive time. We first conducted interviews for a better understanding of the subjective experience of productivity. We found that while the idea of being productive can evoke positive feelings of satisfaction, dealing with unproductive time can be a struggle, negatively affecting people's moods and self-esteem. These findings guided the design and implementation of RU, a tangible device for reflecting on self-care time. Our prototype offers a physical representation of the mainstream productivity mindset and plays with the idea of connecting and charging energy to encourage the user to experience the time considered unproductive as self-care. In a second study, participants used the device for 5 days and our results suggest that the device motivates reflection on activities beyond work and increases awareness of the importance of taking time for self-care.
\end{abstract}

\section{Author Keywords}

Tangible User Interface, TUI, Research Through Design, Productivity, Self-care, Self-Awareness, Interaction Design, Tools

\section{INTRODUCTION}

In an era of hyper-productivity where time is capital, one can identify a deep-seated mindset that constantly pushes people to be more productive. As society subscribes increasingly more to this mindset, even children are taught to think in terms of the productive use of their time [16], and by the time they reach adulthood, they believe that they should strive to optimise for productivity [27]. As a consequence, people struggle to with the tension of balancing work and leisure, as what is considered "unproductive" is de-prioritised [7]. The obsession about avoiding unproductivity results in builtup pressure, leading to stress and anxiety, and potentially culminating in stress fatigue [31].

Productivity is not necessarily bad, evoking feelings of accomplishment and working in a positive reinforcement loop but, if taken to an extreme, it can have damaging health consequences. Setting aside time to breathe and recharge can be deeply beneficial. Taking care of one's mental, physical, and emotional health increases wellbeing, and, in turn, contributes to productivity $[30,21]$. Hence, productivity and self-care are two concepts that go hand-in-hand. As such, helping users strike a balance between both attitudes is an important challenge for interaction designers [34].

While there is a wealth of available tools to support users in self-tracking for improved productivity [40], we found little research on dealing with the other side of this coin. Most available solutions consist of tools that track users' activities at a device or application level, disregarding activities performed beyond computing devices or that do not relate to work-related goals $[1,5,4]$. These technologies are characterized by quantification [43], thus reducing the complexity of life to a numeric representation and ignoring the inherent subjectivity of the lived experience [28].

In this work, we approach the topic of productivity from a different perspective. First, we depart from quantifiable measures, and we tackle the concept from a subjective experience angle. Second, in contrast to common design practices that focus on aiming to right what is wrong or lacking, we use design as a medium to encourage the acceptance of overlooked aspects of life, often considered wrong, embarrassing, or taboo [20, 9]. From a series of interviews, we identified productivity to be perceived as a measure of success, and being unproductive as something shameful and bad. In this research, we aim to encourage users to reflect about a concept that might be understood to be wrong and avoided as something beneficial and an inherent part of everyday life. To do so, we designed and prototyped $R U$ (see Figure 1), a device that motivates users to reflect upon activities that might be considered unproductive and experience them positively as being beneficial for their wellbeing. We believe that re-framing the concept is the first step for a better and more positive understanding of what is popularly considered unproductive. Therefore, in this project, we refer as self-care all activities that fall outside the banner of productivity, but that are nevertheless positive. This intentional re-naming is to avoid the dichotomous understanding of one being good and the other being bad.

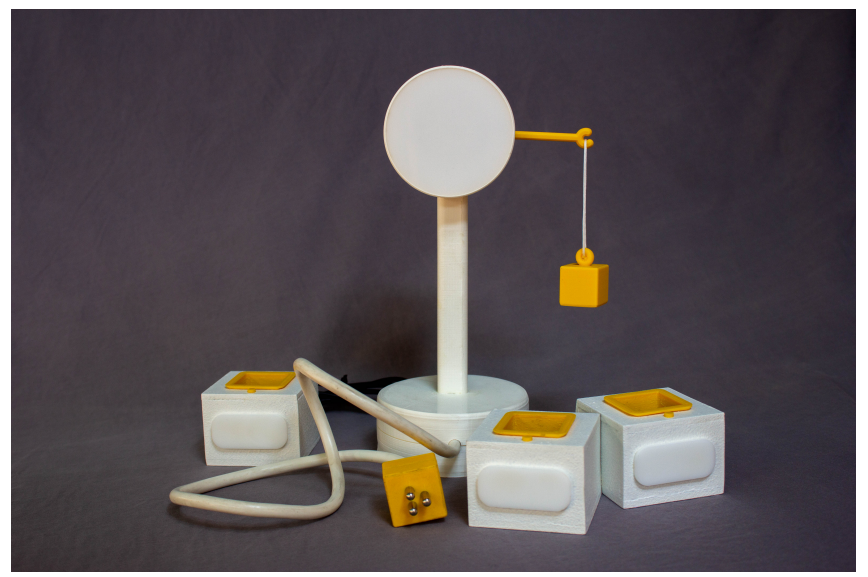

Figure 1. The first working prototype of RU 
Following a Research Through Design (RtD) [44] approach, we used design to explore and produce knowledge for the research and practice communities. This paper reports on the design process of RU, a functional prototype for reflecting on unproductivity and self-care. In this work, we do not aim for the design of a commercial product nor to implement an ultimate and definitive device for behavior change. Instead, we use design practices and qualitative methods to explore the space of unproductivity and self-care with the aim of creating new knowledge about these concepts.

This research comprises of two studies. The first study aimed to explore the design space of productivity. From the 12 interviews we conducted, we gained a better understanding of the experience of productivity, and we uncovered problematic perceptions of unproductivity. Such findings led to the design and implementation of a tangible device for accepting unproductivity. We designed and developed RU and deployed it in a second study for evaluating it in a real-world setting [14, 23]. Our findings support our design intentions of engaging in self-care mindset and demonstrate that the device enhances self-awareness in the benefits and importance of dedicating time to self-care activities. This project demonstrates the opportunity to explore the design space in the periphery of productivity. Our findings motivate and encourage other researchers to pursue the design of tools and technologies for work-life balance from a qualitative perspective, thus prioritizing individual values and emotions.

\section{RELATED WORK}

\section{Productivity trackers}

Tracking productivity has long been an object of interest to researchers and product developers. The key idea behind it is that by improving self-awareness, one can enhance their productivity [30]. When tracking their productivity, the user is given the opportunity to reflect on their behavior, learn from it, and maximise the amount of work they can achieve [29, $12,17]$. As a result, a variety of self-tracking applications and devices have been developed to support users in boosting their productivity by improving their time management (e.g. RescueTime [1], Toggl [5]) or blocking distracting applications (e.g. Focus [4]). Academic researchers have also proposed frameworks that use computing technologies and data analytics as guidelines for users to track, analyze, and improve their productivity $[43,13]$. The most common solutions are systems designed for the digital space in the form of screen-based applications that track the activities the user does on one or multiple digital devices (e.g. computer, tablet, or smartphone). However, digital approaches either limit the tracking to only screen-based activities or force participants to get back to their device to insert their data.

With the increase in adoption of mobile devices and the digitisation of work, data appears to be accessible from anywhere, anytime $[6,38,33]$. The principle behind current systems is that time can be classified as productive or unproductive based on data captured as users carry out their activities. This classification is largely semi-automated, requiring little input from the user. While this seamless approach minimises user effort [32], the system may define a task as unproductive when, in the eyes of the user, it is perceived as productive. For example, whereas reading the news might be unproductive for an engineer, it is a critical aspect of a journalist's work. This disambiguation is often done a priori, with users labelling different applications and websites as productive or unproductive use of their time. Such automation also misses a valuable opportunity for reflection-solutions that aim to quantify human behavior suppress the user with their option to decide upon their actions and discard the values of personal experience and emotion [36].

\section{The subjectivity of productivity}

Little literature is found to study productivity as an experience, using qualitative methods. Kim et al. asked participants to track their personal productivity in a 2-week diary [28]. They concluded productivity to be a state that can take place anytime and anywhere. Besides, while most research studies focus on productivity in the workplace, participants in the study reported a wide range of productive activities beyond typical office work. The authors reported a broad range of tasks and work environments that should be considered to be the focus of productivity.

Tracking productivity can boost productivity. However, ongoing pressure to be productive leads to stress and anxiety, driving to stress fatigue and negatively affecting health outcomes $[41,11]$. This creates a delicate relationship between wellbeing and productivity - while wellbeing positively contributes to productivity, excessive demands on productivity might hinder wellbeing [26, 21]. Therefore, it is crucial to strike an optimal work-life balance [34]: A state of equilibrium in which the demands of both a person's job and personal life are equal [2].

The excessive focus on productivity thinking gives birth to a new lifestyle that opposes it. Anti-productivity challenges productivity thinking, encouraging people to do things that do not yield measurable results, or to do something for the sake of doing it. It also proposes measuring productivity in terms of feelings and emotions rather than what we can produce [18].

As an example of an alternative method to lower the stress of being productive, entrepreneur Mark Andreessen created the concept of the "Anti-ToDo list" [8]. In contrast to the traditional to-do lists where users write tasks and cross them out, the new method consists of only listing the achievements of the day. By implementing this method, the user ends their day with a list of all of the things they accomplished rather than a list of things still left to do. As far as we are aware, there is no literature on the concept, yet research suggests that positive feedback induces rewarding feelings, whereas capturing negative behaviors might increase stress levels on the user [29].

Lastly, at an extreme end of the productivity mindset lies the Chinese concept of $W u$-Wei [6]. The principle stems from the philosophy of Taoism, which emphasizes living in harmony with the Tao ("The Way"). Wu Wei is the balance of the elements both within and outside the body. Although the term translates from Chinese as "do nothing" the actual meaning of the concept is more akin to "effortless action". As such, $W u$ 
Wei implies following natural cycles and not go against nature. The mindset embraces the ups and downs of life, accepting them instead of fighting against them. Flow days refer to high-energy days, whereas ebb days refer to low-energy days. Applied in productivity thinking, the $\mathrm{Wu}$-Wei ideal encourages acknowledging the time of not being productive, rather than enforcing productivity at all times.

\section{Designing for the physical space}

While we found a great number of digital tools, few solutions consider tangible interactions within the physical space to design for productivity and self-care.

Most of the productivity tools that work beyond digital interfaces aim to boost productivity through the improvement of other skills, such as time management, organization, and planning. Bitskit [15] and Daily Stack [24] are task management devices that create physical links to digital information. These applications help individuals to become more aware of their daily workflow and time management through the physical representation of their tasks. Other solutions include systems that minimise interference with the users' daily activities. The Bit Planner [3] is a wall-mounted time and resource planner made of Lego bricks that are synchronized to an online, digital calendar. Any update of the digital calendar is represented in the physical device. The technology engages with the periphery of the user's attention and provides information without being at the center of attention [42].

Physical technologies focused on self-care have resulted in devices that encourage reflection on negative behaviors or situations to gain self-awareness [10]. For example, Nidra [37] targets sleep routines that have been hindered by stress and erratic schedules. The author challenges productivity thinking by encouraging the user to pay more attention to their sleep patterns. In line with this topic, Breakaway [25] explores the design of an ambient display for making positive changes in human behavior, in particular they aim to correct the office worker sitting position.

Concerning stress, the Stressball [39] is a conceptual prototype that explores the emotion of stress from a qualitative perspective. The study challenged the reductionist nature of self-tracking technologies that quantify the human body down to numbers and diagrams. The device resulted in a creative and innovative solution to self-tracking that visualized the users' levels of stress.

So far, we discussed a variety of systems and tools that focus on improving productivity through the design of digital tools that quantify human behavior. While these solutions are mostly based on productivity in a work context, we found literature that suggests productivity to happen anywhere, anytime. These studies use qualitative methods for understanding the inherent subjectivity of productivity and conclude to a wide range of activities beyond the work environment that are considered productive. We also ground our research on alternatives that, at different levels, challenge productivity thinking and insist on the importance of striking an optimal work-life balance. Due to our interest in designing for the physical space, we concluded our related work with solutions for both productivity and self-care that adopt a tangible form. With this paper, we contribute to the exploration through the design of new technologies for re-framing unproductivity. We stepped aside from digital solutions that quantify the human body, and we used qualitative methods for designing a novel prototype that encourages acceptance of a rejected attitude. Our research also adds to the knowledge concerning personal productivity, unproductivity, and self-care. Last, we explored the design space and opportunities with the intention to inspire and motivate other researchers to design new tools for the work-life balance.

\section{STUDY 1: UNDERSTANDING EXPERIENCES OF PRO- DUCTIVITY}

We began our design process by exploring participants' subjective understanding of productivity. We aimed to identify opportunities within the design space of productivity for grounding the design and implementation of a novel prototype.

\section{Study design}

This study consisted of semi-structured interviews about the subjective experience of productivity. We engaged participants in a conversation about how they conceptualise productivity. Through these discussions, we aimed to gain knowledge and understanding of their perceptions and practices in relation to productivity. In these conversations, we guided interviewees through a set of questions that cover the different stages of their experience of productivity. We started by asking them about their own definition of the concept, their feelings and emotions in relation to productivity and unproductivity, and tools and methods they used for improving productivity.

\section{Participants and procedure}

We recruited 12 participants ( 7 men and 5 women) for this first study. Our sample was comprised of participants from different backgrounds, nationalities, and ages (23-69) to include a wide range of perspectives. We interviewed seven people from Spain, one half Spanish half North American, one from India, one from Denmark, one from Italy, and one from Germany. At the time of the interview, two thirds of our participants had lived or were living abroad. Participants had backgrounds in design (3), computer science (2), psychology (1), health (2), business (2), human resources (1), and teaching (1). In this paper, we keep participants' identities anonymous and we refer to them by a numerical identifier (e.g. P9). We note that all selected participants are part of a well educated middle class user group.

\section{Data Analysis}

We conducted all interviews online via Zoom ${ }^{1}$ in either Spanish, Catalan or English as they preferred. All Non-English quotes we report from this study are a translation from the original language.

We used affinity mapping [35] for synthesizing and concluding relevant information from all data. Under the participants' consent, we audio-recorded all interviews for later transcribing them. We first coded all transcriptions, and clustered all

\footnotetext{
${ }^{1}$ https://zoom.us/
} 
information into several ideas and concepts. We iterated on the process of bundling to the point where we had few themes that portray the core findings: definition of productivity, influencing factors, feelings and emotions, the experience of unproductivity, and tools. This procedure helped us to structure our findings and inform the design of our prototype.

\section{Results}

In this section we report on the insights gathered from the interviews. We grouped our findings into fours different subsections that reflect the main aspects discussed in the interviews: the definition of productivity, feelings and emotions, influencing factors and conditions, and tools, techniques and methods.

\section{The understanding of productivity}

Our analysis revealed different conceptualisations of productivity and unproductivity. We started the interviews by asking participants to define productivity. We derived three themes from their responses: goal achievement, time efficiency, perception of progress.

The goal achievement approach consisted of defining a set of objectives to accomplish, normally constrained by a specific time-frame. Thus, this method is closely linked to the second theme time efficiency.

\section{"So for me productivity is achieving whatever goals I set out to do, or achieving small milestones in a project" (P1)}

While this theme is task-driven, participants who focused on using time efficiently were time-driven-meaning participants aimed to seize their time and pursue as much as possible within a given time-frame. Setting the right timings, required self-awareness of the participant's capabilities.

"By productivity I understand to take full advantage of my time." (P3)

"It also requires to know what I'm capable of doing in the given time. Of course, my timing has nothing to do with other people's time" (P2)

On the other hand, participants reported to feel productive when they felt getting closer to their goals. We collected a variety of examples where participants perceived progress: from delivering a project to losing weight. This mindset strongly relates to goal achievement because the user must set a goal to make any progress to it.

"A productive day is when I set certain goals for the day, and I might not have achieved them, but I feel I made some progress on it." (P5)

In conclusion, we identified common themes in how participants conceptualise productivity. The most important parameters relating to how they assess how productive they were included time, tasks, and quality. The first is about setting a time-frame within which productivity will be assessed. The second involves deciding on the tasks and activities to be performed. The third is concerned with the quality of the outcome. For example, users might not feel productive if they accomplished a task within the expected time, but were not satisfied with the result. While time and tasks are quantifiable parameters, quality is a more subjective criterion. "It's a day where I achieve my goals as efficiently as
possible and the best I can" (P10)

\section{The feelings of productivity and unproductivity}

We followed the interviews with a discussion about feelings and emotions. All participants used positive adjectives to describe the feeling of productivity. They identified it with success, satisfaction, fulfillment, and relief. These emotions were positive reinforcing and motivating to keep being productive. Participants called the experience "be in the flow":

\section{"The feeling of having finished tasks is what motivates me the most to do more. Once it starts the flow, it's very smooth. [...] Sounds like positive feedback." (P1)}

We found the concept to appear in several interviews. Participants also mentioned to be a state of high concentration in which time flows:

\section{“...I'm really in the flow, and I forget the time, and that's very nice" (P7)}

But productivity does not always flow. In these situations, participants felt dejected and struggled with the surge of negative emotions. Unproductivity evoked from guilt to frustration, to uselessness. These feelings might reach a point that negatively impacts mood and, in extreme situations, lowers self-esteem.

"When I'm not productive, I'm not in the mood for doing anything. Days I'm unproductive, my self-esteem lowers. I might associate it with my image and things like this. It all builds up, and I start feeling I don't know anything. I feel useless" (P9)

We collected a wide range of different solutions for fighting unproductivity and getting back on track. Some of these include avoiding distractions, taking breaks, switching to other activities, or changing the environment. In any case, participants who instead accepted being unproductive were able to minimise negative feelings and bring calm and relief.

"It can be a very relieving feeling to just put it [the task] aside, for trying out again later. If it doesn't work, then do it another day that you feel with more energy. So it can be good to accept not to be productive." (P7)

“...if I don't do anything is ok. And I think the world is missing this mindset. If you are not productive, it will be $o k$. There's another day, another moment." (P9)

\section{What influences productivity?}

A variety of both external and internal factors affect productivity. The former origins from an external source and the latter involves the state of the user. The first group includes weather, social pressure, and culture. Rainy weather suggested an increase in productivity performance.

"I tend to be more productive when it's raining outside, and I don't worry about missing the sunny weather for example” (P12) 
We also found that productivity increases in productive environments. As such, participants felt more productive when surrounded by other people that seem to be productive. This phenomenon is what we refer to as social influence.

"I like the library or any place where other people studies. [...] I don't get distracted because I see other people working. Being surrounded by productive people helps me to stay focus." (P6)

Participants who lived in different cultures claimed a difference in the society's standards of productivity-the more the cultural impact, the more the difference. For example, in Japan, they have a system for grading productivity in the workplace. High grades give you the right to certain advantages (e.g. salary increase), whereas low grades result in negative consequences (e.g. salary decrease, or get fired). From a Spanish perspective, this is a very objective and strict perception of the concept:

"I have a productivity exam every three months. [...] I then meet my supervisors, and we go through a list of items from which they will grade my productivity. They check on the time we spent, whether we achieved our term goals and the quality of them. They value your communications skills, your teamwork, your learning performance..." (P11)

On the contrary, the Swedish culture allows for more flexibility on productivity outcomes.

"Here in Sweden I feel more relaxed. People here trust each other is responsible for their work, and you don't need to show and demonstrate you have been working." (P9)

Three interviewees started a discussion about the role of society in personal productivity. All three participants blamed society for imposing high productivity standards, building up pressure and stress. According to them, there is the habit of comparing to others, an attitude that has intensified with the use of social media. In most cases, such comparison results in negative feelings:

"You might feel useless if you're not doing 100 different activities" (P3)

“There's this discussion about Shakespeare writing a masterpiece on locked down during the plague of some year, and that we should all now be able to do the same. I'm outraged because you can not set the same productivity standards." (P9)

Regarding internal factors, we identified three main ones: mood, energy, and sleep. All three influence each other in the sense that one being low might induce all being low. Therefore, participants affirmed to be unproductive when feeling down or lazy. On the other hand, to some participants, having loads of energy might not help either to productivity:

"When I have loads of energy I want to do other things that are not sitting on my desk and working" (P7)
On a separate category, we point out the influence of motherhood, which was brought up by one interviewee. The participant reported that becoming a mother was an important catalyst in their productivity thinking. The participant related the experience as a change in priorities and as a gain of selfawareness of time. Wanting to spend more time with the kids implied the need to be more efficient and quickly adapt to new situations:
"It completely changes your capabilities to be productive once you're a mother. I remember spending a lot of time grocery shopping before I had kids. At the moment they were born, I had more interesting and important things to do. I now always buy everything online. It's been like eight years since I started shopping online.” (P4)

\section{Tools, methods and techniques}

In the last part of the interview, we investigated tools used in productivity. We found a variety of tools of all formatsdigital and physical. Their main interest was in systems that supported and enhanced their productivity, and made them reflect on their mistakes and shortcomings. One participant insisted on the difficulty of finding the work-life balance and showed interest in tools that assist in work-life balance.

"I would like a system able to monitor all my activities, and determined my productivity in each of my personal areas. I would like to see if I'm in work-life balance." (P4)

From the interviews, we listed up to 15 different tools and techniques used by participants. We categorized them into tasks and time management tools. As the name suggests, task management tools focus on the organization of tasks and activities. Participants also used these tools to structure and embody their thoughts and ideas. Besides, most of these solutions support the positive reward of checking off a finished task, reinforcing productivity. The most popular tools were Trello ${ }^{2}$, post-its, and checklists.

"It's positive reinforcement. For example, I have my wall full of post-its with ToDo tasks. It might stress me out, so I organize them by importance. To see the number of post-its decrease, so the walls look emptier is very satisfying." (P5)

Time management tools include calendars, agendas, time trackers, and countdowns. The first two support the annotation of important dates and provide a clear overview of their time. Timers and countdowns, instead, monitor time with the aim of enhance efficiency. Nevertheless, most participants rejected these second group of tools for being stressful, obsessive, and distracting. We found only two participants using the Pomodoro Technique [19] on specific situations or tasks:

"At work, we were using time trackers to monitor your time for coding. Before starting to implement the function, you would press start, the time would start ticking, and you wouldn't stop it until you finish. It generates a lot of anxiety. [...] I was checking the timer instead of being focused on coding." (P11)

${ }^{2}$ https://trello.com 
"The Pomodoro Technique helps me when I have tasks of coding that should be quick. I've also used it in brainstorming sessions that need to be dynamic. I only use it on tasks that need to be done in a relatively short time." (P5)

\section{Discussion}

This study of 12 participants confirmed the inherent subjectivity of productivity. Although participants shared similar notions of what productivity is - the experience of getting things done within a time frame- their specific perspectives somewhat differed. Productivity was generally related to office work, yet participants included other types of activities (e.g. sports) as well. We identified a pattern among these other activities - they tended to be driven by outcomes, a deadline, or a sense of duty (e.g. cleaning, grocery shopping). Activities like reading or watching TV did not contribute to productivity unless having a work objective. On the other end, participants attached the label of "unproductive" to passive activities that required little or no interaction, or lack of a clear purpose (e.g. be on social media, taking a nap).

Our findings suggested a need for an improved conceptualisation of productivity, which leads to negative feelings when out of control. However, there is a variety of factors and conditions, both internal and external, that affect productivity. While people might have a certain level of control over some of them (e.g. sleep, mood), others fall out of their control (e.g. weather). Participants also commented on the role of society in evaluation of productivity. Assessing productivity was, in most cases, also influenced by mainstream productivity thinking. Society setting high standards of productivity and comparing oneself to others results in built-up pressure and stress. These standards vary from culture to culture, meaning there is a correlation between culture and productivity. We conclude that productivity is a dynamic phenomenon, adapting to new situations and environments like in the case of motherhood.

All these circumstances might generate frustration in moments of low productivity. Being in a state of flow-highly focused and productive- - evokes positive feelings of success, satisfaction, and pride. It works as positive reinforcement and motivation to stay on track. On the contrary, unproductivity can have the opposite effect. The negative feelings might reach a point of impacting mood and even self-esteem. While most participants acknowledged fighting against these situations, those who went through a process of accepting their circumstances were better equipped to cope with negative emotions.

The results from this study and related work motivated us to (1) reject the quantification of productivity and use qualitative methods for exploring the experience; (2) reflect on the negative implications of a society in mainstream productivity thinking; (3) focus on the struggle of dealing with the counter effects of productivity, and enhance the positive consequences of accepting unproductivity; and (4) overcome the limitations of digital solutions - that monitor productivity on a device level or force the user to input the data manuallyand explore the potential of designing Tangible User Interfaces (TUIs) in this context. We uncovered a variety of design opportunities, from which we discarded all solutions focused on improving, enhancing, or boosting productivity due to the amount of work already done $[1,5,4]$. Instead, the scope of this research is to design a tangible device that embodies mainstream productivity thinking and encourages users to embrace and accept unproductivity rather than fighting against it. In short, we aim to re-frame the concept of unproductivity to a positive self-care experience.

\section{RU: DESIGN AND IMPLEMENTATION}

Based on the findings from our first study, we started an iterative process of sketching, lo-fi prototyping, material exploration, and role playing to evaluate and elaborate on different the different ideas. We departed from the concept of work-life balance, and each iteration helped us gathering feedback that would shape the next prototype (see Appendix D and E).

This process led to the implementation of $R U$, a device for reflecting upon and accepting the time and energy spent on activities that might be considered unproductive, but are essential for maintaining the user's wellbeing. We named the system $\mathrm{RU}$ after the old Swedish word ro, which is pronounced $r u$ and means peace and calm. At the same time, RU can be read as the English acronym of "Are You", prompting the question "Are you OK?".

The metaphor that guided the design or RU was that selfcare is the energy that allows us to carry the weight of our work life. We designed the system as a device that makes these concepts tangible while giving continuous feedback as an ambient display [42]. As a symbol for the weight of the productivity mindset, the device has an arm that carries a weight. Throughout the day the arm becomes "tired", slowly and almost imperceptibly lowering the weight up to a point where it is dropped.

To prevent the weight from dropping, the user must "charge" the device. This can be achieved by plugging it into one of the sockets in a set of blocks provided to the user. Each block represents a self-care activity defined by the user. The user can also set a strength for that activity by turning a knob in the corresponding block, depending on how they conceptualise their contribution to their own self-care. For example, the user might have one socket for the activity "play tennis" set to maximum importance because they feel that is a crucial activity for their wellbeing. Then, every time the user practices the sport, they would connect RU to the socket for charging RU.

RU continuously discharges over time. Every 25 minutes, the hook rotates two degrees clockwise. The movement is small and smooth, making the change only perceptible after a long time. It takes around 16 hours for the hook to travel from one end to the other. These are the total of waking hours in a day, taking into account that 8 hours of sleep is an often recommended amount of sleep time [22].

RU charges when connecting it into a socket (see Figure 2). Then, the hook rotates anticlockwise as much as the value given to the socket. This value is proportional to the charge capacity - the bigger the value, the more the charge capacity and the longer is the hook ticking anticlockwise. When 
charging, the user must push down the plug until fully charged, which can take up to 5 seconds. Otherwise, the charge is interrupted at half load. This way, we ensure the user does not leave connected the plug with the intention of continuously charging. When connected, RU's face lights up and its brightness increases as it charges. The light color switches from yellow to green, which indicates full charge.

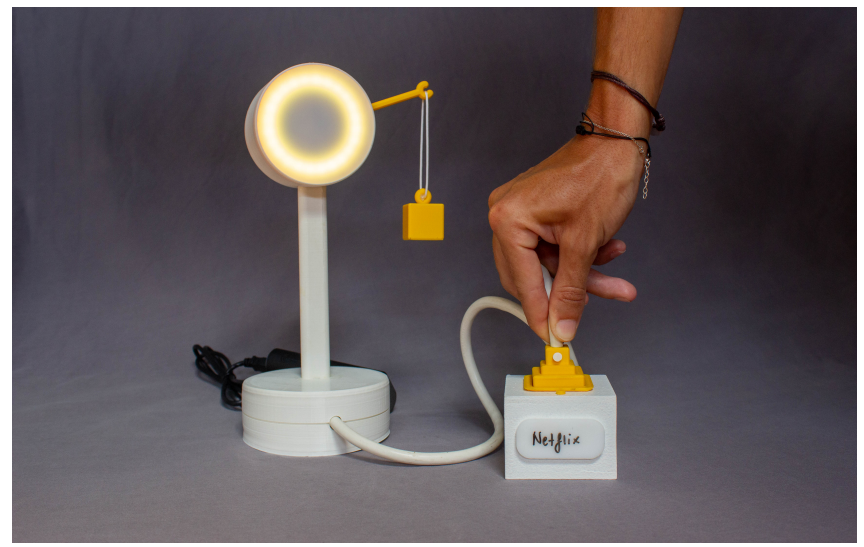

Figure 2. The interaction for charging RU to the socket with the activity "Netflix" assigned to it

The hook only reaches its highest position when the user has plugged several sockets in a relatively short time. Nevertheless, if the user does not connect RU to any activity for a long time, the hook keeps ticking clockwise to the point of reaching the lower position. This increases the chance that the hanging object drops and fall out the hook. Through this subtle interaction, we notify the user of the need for self-care activities and the risk of possible stress fatigue.

RU's aesthetics resembles a scale, thus reflecting the importance of work-life balance. By charging and discharging RU, the user is automatically committing to their self-care. RU demands little effort but continuous use, reminding the user to be consistent with their self-care activities.

\section{Hardware}

RU has two different parts: the main station, and three sockets.
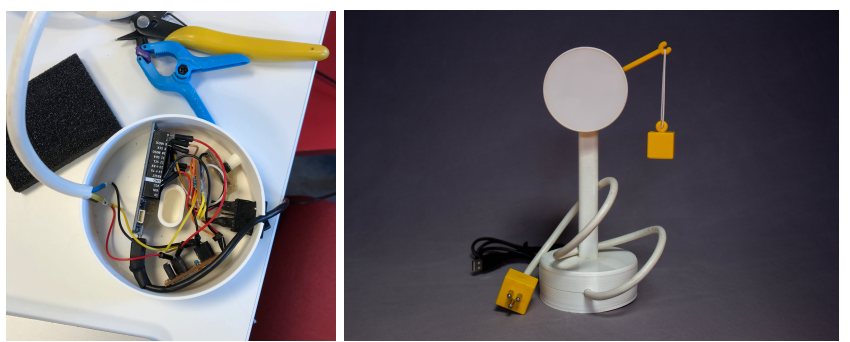

Figure 3. The main station of RU. On the left, the base with all the electronic components in it. On the right, the final design with all parts assembled

The main station (see Figure 3) consists of the base, at the bottom, and the face, at the top. The stem joins both parts. From the base, a yellow plug comes out from the front. We also added an on/off switch and reset button at the back of the base. The face has a built-in light system and a hook coming out from the right side. From the hook, there is a hanging cube that represents weight. Both the hook and the weight rotate clock- and anticlockwise up to 20 degrees from the balanced position, when the hook is neither tilted upwards nor downwards. When resetting RU, or turning it on for the first time, the hook resets to the balanced position.

The sockets (see Figure 4) are wooden blocks that represent the different self-care activities assigned by the user. Each socket has a whiteboard at the front face for writing down the activities' name. In the back, we added a knob for the user to indicate the importance of that corresponding box-a higher value means more importance of the activity which translates to more charging capacity.
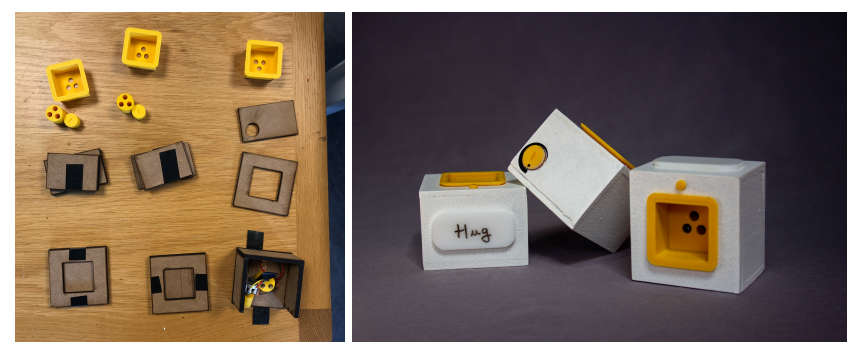

Figure 4. The three sockets. On the left, a picture of each socket disassembled. On the right, the final prototype

From a technical perspective, the device consists of four main components: the light, the hook, the plug, and the socket. All of them connect to an Arduino MKR1000 WIFI ${ }^{3}$ that controls all the logic of the system. The micro-controller is held in the base of the main station (see Figure 3, left). For the lighting, we used a 24 LEDs NeoPixel Ring ${ }^{4}$ that supports different colors and brightness. The hook is attached to a mini servo that rotates clock- and anticlockwise. The servo is limited, with software, to rotate up to an angle of 40 degrees from one end to the other. Last, the plug does not have a standard regulated design. Its design is specific to the device, meaning that only fits to the outlet in the sockets. We recycled the cable and the pins of regular plugs to implement a customized plug with three pins: one for power, the other for ground, and the third is data. The shell was 3D modeled for 3D printing. When plugged to the socket, each pin of the plug connects to the corresponding pin of the potentiometer inside the socket. The potentiometer allows the user to change the value of the socket. The sockets are powerless blocks and when RU is plugged into one, the electronic circuit closes, and the micro-controller reads the value of the potentiometer resistance. To ensure this connection, we implemented a system made of springs for a better current conduction from the main station to the potentiometer.

The device is powered using a power bank that connects to the black USB port sticking out from the back of the base.

Concerning materials, $\mathrm{RU}$ is made of strong plastic and wood. Throughout the design process, we explored different materials, but plastic and wood resulted in the most moldable materials for achieving the desired form of the artefact. We

\footnotetext{
${ }^{3}$ https://store.arduino.cc/arduino-mkr1000-wifi

${ }^{4}$ https://www.adafruit.com/product/1586
} 
designed and modeled the main station and some elements of the sockets for 3D printing. We used ABS yellow and PLA white for 3D printing materials. All components were printed separately and later assembled together. Finally, the sockets are made of wood painted in white to fit the aesthetics of the device (see Figure 4, left).

\section{STUDY 2: DEPLOYMENT AND EVALUATION OF RU}

The second study focused on the deployment and evaluation of RU in a real-world setting. In this study, we aimed to understand how a tangible interface can re-frame the concept of unproductivity. From the outcomes of the study, we also expected to learn new meaningful concepts regarding selfcare, to explore new ways of interacting with the device, and to test the device from an technical perspective. Last, we also aim to gather inspiration on how to design new kinds of technology [23].

\section{Study design}

We decided for an in-the-wild [14] deployment of a technological probe where the participant takes the device home to use it without the supervision of a researcher. Each study had a duration of five days. We considered this time-frame to be appropriate for the user to explore RU, and for the research team to detect any changes in the user's experience of unproductivity.

We provided each participant with a complete kit with everything needed for the use of the system (see Figure 5). The kit included RU, three sockets, the diary probe, the instructions of use, one power banks, a whiteboard marker and eraser. We asked participants to complete a diary throughout the study. The diary had four parts: the location, the sockets, the daily report, notes, and comments. In the first section, the participant described where they placed RU in their environment. In the second section, we asked the participant to keep track of all the changes applied to the different sockets and knobs. This part included activities and importance assigned, the reason for the choice, and the date of the change. In the third section, we aimed to understand the daily use of RU. In this part, we asked the participant to summarize their feelings of that day, $\log$ in the activities used for charging RU, and indicate if the hanging weight dropped at any point during the day. Last, the diary had space for the user to take notes, comments, or feedback.
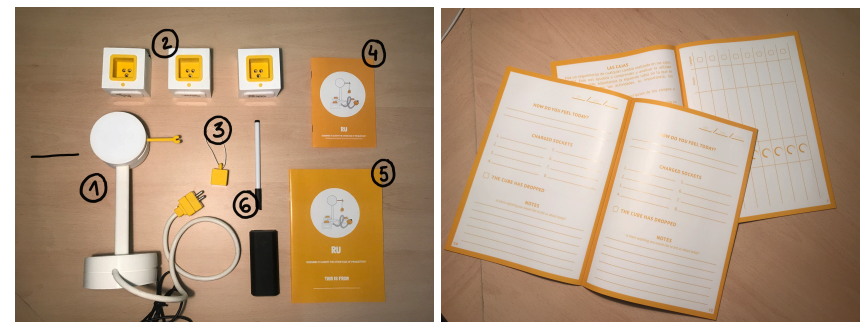

Figure 5. The evaluation kit and diary probe: (1) RU, (2) three sockets, (3) weight, (4) instructions of use, (5) diary probe, (6) whiteboard marker and power bank

\section{Participants and procedure}

We recruited five participants (P13-P17) from different backgrounds, ages (25-59), and gender (4 females and 1 male) for testing and evaluating RU. All participants were office workers with positions in marketing manager (1), study coordinator (1), business manager (1), psychologist (1), and teacher (1). Participants are all Spanish and lived in the same geographical area (Region of Barcelona, Spain) by the time of the study.

At the start of the study, we gave participants the details of the research and specific instructions on how the test would proceed. We introduced the device and carefully explained how it worked. We also provided the instructions in written form. We emphasized the free nature of certain parts of the study, such as designating activities to the sockets, and the importance given to them, which were left open for participants to assign whichever way they wanted. Following, we guided them through the different tasks to perform during the study. We explained how to complete the diary, which also contained a written description of each section. Lastly, we provided them with the contact information of the principal researcher for any problems that might arise during the study. After the evaluation period, we met the participant again for picking up the device and conducting the final interview about their experience using the device.

We informed participants of the anonymity of their responses. In this paper, we refer to participants with a numerical identifier (e.g. P16).

\section{Data Analysis}

In this study, we also followed affinity mapping [35] for synthesizing and structuring all collected data. We conducted all interviews onsite in Spanish, translating to English any quote we report. At the start of the study, we asked the participant for their consent to audio-recording the interview. We proceed by transcribing all interviews and coding them and the diaries. We then grouped the listed codes into concepts and ideas, iterating the process to reflecting the core findings.

\section{Results}

In this section, we report on the results obtained from the interviews and diaries. We first present the findings regarding the overall experience of using RU as a tool that motivates reflection on self-care time. We then follow with the interpretation and meaning given to connecting and charging RU. We last present meaningful information regarding self-care activities, and the criteria to assess them.

Each study, except for one, had a duration of five days. In the case of P16, the participant encountered a technical problem halfway through the study. After fixing the problem, we decided to extend the study's duration by one more day. Hence, the evaluation period with P16 lasted a total of six days. No other technical issues arose during the study. In the interviews, some of the participants mentioned having gone through an exploratory phase for understanding the device functionalities before making full use of it. They all reported the device to be very intuitive and easy to use, and to not feel overwhelmed by the tasks and interactions of RU. 


\section{A tool for reflection}

Participants described the experience of using RU as positive, helpful, motivating, and innovative. RU helped them to engage in reflecting on activities that fall outside the work environment and contribute to self-care, increasing self-awareness on the importance of taking time off productivity:

"It's super interesting the fact that makes you reflect. It makes you think what I do that might be productive, but it's considered unproductive. [...] Sometimes we give more importance to being productive, and we feel the need of doing so many things, but we have to do activities that contribute to your wellbeing." (P14)

"It's been a very positive experience. In the beginning, I thought it would be boring because of the writing in the diary, but it turned out to be a tool that helped me to take more time for myself." (P15)

Participants interacted with RU mostly at the end of the day. In some cases, they would charge RU right after performing the activity, though it was only possible if they were at home where RU was. Not being able to connect RU at any time yield to the opportunity for reflecting on all the activities of the day. One participant also elaborated on the idea of productive activities is time you invested in others, whereas self-care time is for oneself:

"I would do it before bedtime. For me, it was a moment to stop and think: what have I done today that's been good for me?" (P13)

Another participant insisted on how productivity thinking blinds them from self-care activities. The participant used the concept "generalization" to describe situations of little awareness of the good experiences because these are masked in a negative feeling produced by productivity thinking:

"It helped me to be more aware that I have to take time for myself. Sometimes you feel you only work, but if you stop, you see that there are moments in which we do nothing, and it's good because we need them. Yet, we don't stop. [...] There are always moments throughout the day that we disconnect from productivity." (P16)

Participants translated the interaction of connecting and charging RU to themselves, feeling as if charging their wellbeing:

"I had the feeling I was charging batteries. [...] It helped me to charge my batteries.” (P15)

"It was like I was doing it to me, as if I was connecting and charging myself." (P13)

Last, regarding where to locate RU, participants opted for a place of high visibility to them, and the device would work as a reminder. Participants also chose a desktop or similar for a better comfort when writing in the diary.

"I chose to put it in my nightstand because it's my 'nonworking spot', my place for reflection. Besides, it gives me privacy, and it [RU] won't be exposed to the rest of the family." (P15)

\section{The interpretation of connecting and charging RU}

Given the duration of the study, all participants except for one held and used RU during weekdays and weekend. Instead, P15 explored the device from Monday to Friday. From analyzing the diaries, we identified a noticeable difference between weekdays and weekend concerning activities and interactions with RU. Participants interacted differently according to the day of the week. In the weekend, participants registered more activities, so the hook tended to be tilted upwards and the weight not to fall. One participant mentioned that they managed to keep the device so charged that the hanging cube ended up falling in the opposite direction. The participant reported this as a very positive finding, as it rewarded them for spending time on self-care:

"The day I spent all day with my daughter, [...] and charged to the highest position. That was amazing. It was very positive feedback, and I thought: I achieved it, I reached the top. It was motivating and super satisfying." (P16)

On the other hand, during work days, most of the participants spent a long time without interacting with RU and reported to have less time for self-care activities. For a few participants, not to charge RU in a long period turned out to be inconvenient. To find the weight dropped frequently evoked a bit of frustration. Yet, all participants were, at different levels, triggered by it and motivated to engage in self-care:

"It was frustrating because it was falling every day. I would spend 8 hours working, and it would fall. Despite that, it was positive. [...] It helped me. It was motivating to challenge myself. It [RU] pushed me to do more activities, so the cube doesn't fall.” (P15)

"I was expecting it [to see the cube on the floor] because I would spend all day at the office. Anyway, it was like a representation that I should spend more time in self-care activities. It was a bit sad [to find it that way], but then I would charge all the activities and think the day wasn't that bad." (P13)

Nevertheless, two participants suggested two different solutions to overcome this problem. On the one hand, to increase the time for the hook to reach the bottom position. There are two ways of achieving this: increasing the time interval; or setting the initial position to the top rather than the middle/balanced. On the other hand, participants recommended complementing the system with an app or additional device that allowed them to charge RU anywhere at anytime, allowing interaction with RU throughout the day rather than connecting all the activities at once.

\section{Self-care activities and how to assign them}

We found two methods for the use of the sockets: the flexible and fixed approach. The former consists of assigning an activity per socket, having three simultaneously. In case the participant wanted to add a new activity, they would erase what the socket's whiteboard has written and write down the new activity's name. On the contrary, in the fixed approach, the participants gave each socket an importance value-high, medium, and low_-and assigned multiple activities to one 
same socket, based on the level of importance. Having fixed activities worked as a reminder and pushed them to take time for performing the selected activities. Besides, this approach afforded fewer changes and a higher number of activities at the same time.

Despite these two different ways of interacting with the sockets, all participants had the same criteria for determining the importance of an activity. The value of the socket was given based on the feeling resulting from doing the activity. The higher the contribution to self-care, the higher the value of the socket. Participants illustrated the process of giving value with examples:

"To the activity music, I updated the importance. Maybe at one time, it is of medium importance, and at another moment, I spend the afternoon listening to music, dancing, and singing. Then I'm super happy, so I give it [the activity] a higher value." (P15)

In one case, the participant stated that it was confusing to regulate the importance in certain situations and activities.

"I don't know up to which point doing nothing contributes to self-care. If I was tired and I just want to watch TV, should I give it low value because doesn't contribute or high because I'm super tired? Instead, I was sure when I was going for a walk. I do it because I need to go out, take some fresh air, and then I would give it a high importance." (P17)

Concerning activities, participants registered up to 50 entries in the activity registration section of the diary. From all of them, we identified a total of 32 different activities, which we grouped into six categories based on the reasoning for choosing the activity. In some cases, one same activity was experienced differently, thus falling into two different categories. Below, we list the different groups with a short description and examples:

- Relax: The majority of the total registered activities fall into this category. This group covers a wide range of different activities that help the user relax. These activities were given medium and high importance in fifty-fifty. Examples: reading, listening to music, watching TV, playing games...

- Disconnect: This category covers activities that induced a mental break from productivity thinking and their routines. If the participant got to disconnect, the activity was of great value for them. Hence, most of them had high importance. Examples: reading, going to the beach, hanging out with friends...

- Rest: This category includes all activities with a passive interaction-the participant's interaction was little or none, and lacked of intended goal. We divided them into two subgroups: sleeping and do-nothing. The former is the time spent sleeping or similar (e.g. taking a nap, snoozing the alarm). The latter includes activities with no clear purpose (e.g. scrolling on the phone). All rest activities contributed medium-low to the participant's self-care.
- Positive Energy: This category covers a wide range of different activities the participant performed just for the sake of having fun and enjoying the activity. These activities result in a positive change of mood. Participants described the feeling as "recharging your batteries" (P13). Hence, participants assigned medium-high importance to all activities. Examples: going out for dinner, watching Netflix, or walking...

- Care: Within this group, all activities in which the participant took care of themselves or another object/ living being. Participants considered these activities of mediumhigh importance. Examples: petting your dog, face cleaning, gardening, sport...

- Strengthen relationships: Spending time with family and friends was of great importance for participants. All these activities had a social component, and their respective socket was set to the maximum value. In these activities, what mattered to participants is with who they share the time rather than what they did. Examples: playing board games, going to the beach with friends...

One participant insisted on the difficulty of selecting self-care activities, choosing to only register deliberate activities.

"I didn't add the times I was working and I would check twitter. This was not intentional, I didn't need a break." (P15)

The same participant claimed a distinction between activities and in-activities. The first kind is where the user was actively involved in the activity. On the contrary, in-activities was the time when the participant did not interact.

"I had to connect and turn on the light anytime I wanted to disconnect and pursue and activity that is not productive, but not an in-activity. That's why I hesitate on registering $T V$, because my brain activity is very low." (P17)

\section{Discussion}

This study concluded with promising results suggesting that the use of RU resulted in an innovative and positive experience for participants and that its design was successful in terms of aesthetics, functionality, and meaning. First, the device presented a clean, minimal, and creative design that pleased participants. Second, RU offered the opportunity to focus on the experience of self-care, which was considered inspiring by our participants. Also, the results confirmed our intentions of the value in designing a tangible device for reframing unproductivity. Participants reflected upon activities commonly understood as unproductive, and instead registered them as self-care activities. Third, RU used its design to embody mainstream productivity thinking. The study brought up new research questions regarding the understanding of selfcare in other cultures and work settings and provide insightful feedback for improvements in further design iterations.

\section{The experience of $R U$}

We found that our device encouraged and motivated reflection about self-care activities, and also raised awareness of the 
benefits and importance of dedicating time to self-care. We designed RU to embody the mainstream productivity thinking, which turned out to be understood and perceived accordingly by participants. The idea of connecting and charging self-care represented the idea of "recharging batteries" that symbolize energy. Participants associated the movement of the hook with personal satisfaction. To reach the top position meant success and evoked positive feelings, whereas the bottom position prompted frustration. With the idea of dropping the hanging weight after not charging RU, we intended to represent productivity stress fatigue. Participants did not interpret the metaphor as expected, yet they understood it as a situation to avoid, triggering them a change.

Testing and evaluating RU on a real-world setting uncovered different ways of assessing self-care activities, and assigning them to the sockets. We found participants using fixed sockets-having the same activities throughout all the study, and flexible sockets-adjusting their sockets almost everyday. We identified the advantages and disadvantages of each method. The fixed socket method follows a mental process of selecting just a few self-care activities and establishing them once in the early stages of the study. This process induces personal reflection, requiring self-knowledge for choosing the right activities. Focusing on a few activities, motivated and challenged the participant to perform them. The method also reduced workload for the participant, minimizing changes in sockets. The main disadvantage of using fixed sockets is the limited spectrum of activities to just a few, automatically discarding other possible self-care activities.

On the other hand, working with flexible sockets afforded a great variety of possibilities for charging RU. However, this added work for the user in assigning activities. Yet, this was the most common approach among participants. The method worked as a medium for reflecting on day-to-day activities, offering a bigger picture of their daily self-care.

Each method influences differently on frustration levels. Having a wider range of options to plug in, increased the possibility of charging more and maintaining the hook tilted upwards. This resulted in less frustration. Instead, participant who used fixed sockets narrowed down their options to just a few, feeling more frustrated.

We also noticed a correlation between the day of the week and the frequency of interaction. As we expected, there were more activities registered during the weekend than on weekdays. The weekend provided more time for self-care, charging more activities and more often. On the contrary, weekdays seemed ruled by routines. All participants were workers, having labor schedules of around 5-8hours straight and spending most of the day away from RU. The lack of interaction with RU due to their schedules and routines generated frustration at finding the weight dropped after work.

\section{The self-care mindset}

The outcomes of the study also contributed to our knowledge about the experience of unproductivity and self-care. The results show the consequences of mainstream productivity thinking - people not being aware of their wellbeing and in- vesting little time on self-care. The tool we designed carefully pushed participants to the self-care mindset, making them more self-aware of it. We also found unclear criteria to determine whether it is self-care or not. Participants were unclear which kind of activities contribute to self-care. This resulted in either considering all activities that fall out of the work context, or selecting just activities that are commonly categorized as wellbeing and self-care (e.g. sport, reading...).

Our findings on the different activities show that all evoke positive feelings and emotions, meaning there is a common belief of self-care as a positive concept. For a better understanding, we decided to group all registered activities by feelings. We considered other criteria for categorizing the activities (e.g. similarities or its nature), but we concluded more meaningful information from studying them from a qualitative approach.

In general, we noted a higher registration of active activities rather than passive ones. Just one participant mentioned this distinction in the interviews, but we found the same pattern on all studies. Active activities include activities driven by a purpose, where the participant was actively interacting in the activity (e.g. gardening, hanging out with friends...). On the other hand, in passive activities - also referred to as inactivities - the interaction of the user is low or in-existent, and there is no determined objective (e.g. scrolling on the phone, lying on the sofa, watching TV...). According to the interviews from study 1 , the latter group include activities that people associated with unproductivity. The use of passive activities to charge RU implied participants perceiving them positively, and experiencing as self-care. We also stress the contributions of a social component to self-care activities. We identified it over different categories-Strengthen Relationships, Relax, Positive Energy, and Disconnect-, affecting the experience in different ways depending on the activity and the participant. We also point out the translation of taking care of other living beings to one's self-care. Results revealed that being actively looking after another living organism impacts on self-care.

\section{Further work in future design iterations}

We identified further improvements for the next iterations of the prototype. We got inspired by the recommendations of participants, and we propose several upgrades. We acknowledge adding more time for the weight to drop by changing the start position from the middle to the top, which will double the starting time from 8 to 16 hours. We also consider the implementation of a smart system that adapts the timings to the user's schedule which could be manually inputted by the user or with a semi-automated component. We question whether manual input would prompt reflection and self-awareness on their time, schedules, and routines.

We also observed the inconvenience of switching off the device overnight, stopping the hook from ticking. In some cases, they forgot and started the next day with a very unbalanced position and the weight dropped. We suggest adding a "sleep mode" where the system would automatically stop functioning overnight. Last, we also consider the suggestion made by one participant in complimenting the current system with an app or an additional device for charging RU from anywhere anytime. 


\section{Limitations}

In this study we limited our population to five office workers. We bring up the opportunity of further evaluation to explore the impact of routines on self-care time and unproductivity by recruiting participants with different profiles such as homeoffice workers, students, or even people on a vacation term. We also limited the study to a user group of well educated middle class people. Targeting Including other user groups of other economic and education levels might result in different perceptive and understanding of productivity, unproductivity, and self-care. Another limitation was to participants within a closer geographical area, sharing the same culture and lifestyle. In Study 1, we reported the influence of cultural on productivity thinking (e.g. Japan vs. Sweden), questioning now the existence of any possible cultural constraints on self-care activities. Further work could complement the insights from this study with results that include groups from different geographic areas.

\section{DISCUSSION}

\section{Using design for engaging in reflection}

In this paper, we explore the design of new technologies that encourage a positive notion of unproductivity. We stepped out of all solutions that aim to right the wrong, and instead, we focused on designing for re-framing unproductivity. One of our research questions was to validate whether our design leads to a positive change in the perception of unproductivity, a concept highly avoided among people. In short, we wanted to design a tangible device that helped people to start experiencing the time being unproductive as time dedicated to self-care and wellbeing.

The results of the second study corroborated our design intentions to an extent. In Study 1, we found a general understanding of passive activities as unproductive. The use of passive activities for charging RU suggests that participants accepted them as self-care. In this case, we achieved our goals of re-framing the concept of unproductivity.

On the other hand, our findings strongly support the design of a tool for engaging in reflection. RU has demonstrated to give space for reflecting on productive, unproductive, and self-care time. Our prototype enhanced self-awareness on the benefits of self-care and the importance of it, acquiring a reminder function for taking time aside from productivity thinking. The device embodied the productivity mindset and motivated the user to challenge it. Overall, the device guided users through an inspiring and positive experience of reflection and self-knowledge.

We take this discussion to bring up new directions for further research. We propose a follow-up study to evaluate the participant's self-care mindset after having used RU. We wonder if the implications of RU are strong enough for the participant to still reflect on self-care activities after a while. We also question whether the same design can be generalized to different topics by translating the current metaphor to a different one. For instance, it might be of interest evaluating the implications of this device for productivity.

\section{Productivity, unproductivity and self-care}

While the first study focused on the experience of productivity, the second study resulted, among other findings, on knowledge about self-care. Our findings suggest a clear distinction between three different experiences: productivity, unproductivity, and self-care. Productivity is the state of getting things done. In the first study, we uncovered several factors that influenced productivity and led to unproductivity. Hence, unproductivity occurs when there is a desire or need to be productive but no success. Last, self-care comprises all activities that contribute to one's wellbeing. Our second study illustrated the subjective criteria to assess these activities. A same activity feels unproductive or self-care based on the interest in being productive. For example, checking out social media is perceived as self-care or distraction according to the aim of being productive.

From the first round of interviews, we learned that productivity produces very contrary feelings. You can be at the top, feeling successful and satisfied, or at the bottom wrapped in negative emotions. Instead, self-care activities evoked positive emotions that contributed to wellbeing. Productivity thinking craves for more production and a busy lifestyle, whereas the self-care mindset surrounds people in a calmer and peaceful mindset. Self-care slows down the high-speed lifestyle. These findings confirm our beliefs, also grounded by literature, about the importance of finding a balance between the two attitudes to avoid stress fatigue.

We elaborate on the idea that self-care is a more abstract concept where feelings prevail whereas outcomes drive productivity making it more measurable. Despite being also a subjective experience, participants assessed their productivity based on quantifiable parameters (time and tasks). For the same reason, current systems quantify the experience. We observed a strong association of productivity with work-context activities, helping participants to differentiate productivity from not productivity.

We emphasized the unclear criteria to determine whether an activity is self-care. From the second study, we found participants that contemplated all activities that fell out of the work environment, or participants that associated self-care to the time invested in oneself. The conflict arises when considering active (e.g. sport) and passive activities_or in-active (e.g. lay on the sofa, be on social media). While participants clearly identified the former to self-care, in some cases, they found it confusing to consider the latter as well. We could have cleared out their confusion by listing all possible activities, but we risked a potential bias on the participants decisions. Instead, giving the participant complete freedom stressed them to reflect on their time and reduced any shortcut on their mental process. We went away from implementing a system that decides for the user and explored the design of a tool for assisting users in making sense of their behavior. With the prototype $\mathrm{RU}$, we opened up a space for reflecting on self-care time.

\section{ETHICS}

Due to the nature of this research, which involved people alien to the research team, we were required to apply for ethics approval. This was submitted and approved by an ethics 
committee in Psychology Health and Applied Sciences from the University of Melbourne. The application was assigned to the id 1853072, and its maximum expiry date is December 2023. We note that this approval was valid for both studies of this project, user interviews, and device evaluation.

\section{CONCLUSION}

The tendency of quantifying subjective experiences like productivity drew our attention to approaching the topic from a qualitative perspective. We first conducted 12 interviews to understand the subjective perception of personal productivity and to identify the tools, methods, and techniques currently used for improving it. Our findings confirm the subjective nature of productivity and reveal insights about the experience. We report on the counter effects of productivity, which are neglected by society. The feeling of being productive is commonly associated with success and satisfaction, whereas unproductivity is considered a failure. However, participants who learned to accept the unproductive time brought relief and calm to their wellbeing. From this finding, we started an exploration through design to build a device for re-framing unproductivity - meaning the acceptance and change of perception from negative to a positive experience. We then created RU, a tangible device that embodies mainstream productivity thinking and plays with the idea of connecting and charging to motivate reflection on self-care. We held a second study with 5 participants for validating our design and evaluating the device in a real-world setting. RU motivated and encouraged reflection on self-care activities, enhanced self-awareness of the benefits and importance of self-care, and challenged our participants to step away from a productive mindset and dedicate time to self-care. Our research work explored the use of design in the creation of new technologies for re-framing unproductivity and contributes, through a qualitative approach, to the knowledge in understanding the personal experiences of productivity, unproductivity, and self-care. Overall, we opened up a new space for further research, and we aim to inspire other researchers to depart from the quantification of human behavior and explore new technologies that engage in user experiences.

\section{REFERENCES}

[1] 2007. RescueTime. (2007). http://rescuetime.com/

[2] 2012. Work Life Balance. (2012). https://wordspy. com/index. php?word=work-life-balance

[3] 2013. Bit Planner. (Nov 2013). http://www. bit-planner.com/

[4] 2015. Website Blocker and App Blocker for Mac Focus. (2015). https://heyfocus. com/

[5] 2018. Free Time Tracking Software. (2018). https://toggl.com/

[6] Gregory D Abowd and Elizabeth D Mynatt. 2000. Charting past, present, and future research in ubiquitous computing. ACM Transactions on Computer-Human Interaction (TOCHI) 7, 1 (2000), 29-58.

[7] David Allen. 2015. Getting things done: The art of stress-free productivity. Penguin.
[8] Mark Andreessen. 2007. Guide to Personal Productivity. (2007). https:

//pmarchive.com/guide_to_personal_productivity.html

[9] Jeffrey Bardzell and Shaowen Bardzell. 2013. What is" critical" about critical design?. In Proceedings of the SIGCHI conference on human factors in computing systems. 3297-3306.

[10] Giulio Barresi. 2018. Tools For Connected Humans. (2018). http://connectedhumans.tools/

[11] Joel Brockner. 1985. The relation of trait self-esteem and positive inequity to productivity. Journal of Personality 53, 4 (1985), 517-529.

[12] Jonathan J Cadiz, Gina Venolia, Gavin Jancke, and Anoop Gupta. 2002. Designing and deploying an information awareness interface. In Proceedings of the 2002 ACM conference on Computer supported cooperative work. 314-323.

[13] Ryder Carroll. 2018. The Bullet Journal Method: Track the Past, Order the Present, Design the Future. Portfolio.

[14] Alan Chamberlain, Andy Crabtree, Tom Rodden, Matt Jones, and Yvonne Rogers. 2012. Research in the Wild: Understanding 'in the Wild' Approaches to Design and Development. In Proceedings of the Designing Interactive Systems Conference (DIS '12). Association for Computing Machinery, New York, NY, USA, 795-796. DOI: http://dx.doi.org/10.1145/2317956.2318078

[15] Sally Chan. 2013. Bitskit. (2013). https://sallychan.ca/bitskit

[16] Christine Chin. 2007. Teacher questioning in science classrooms: Approaches that stimulate productive thinking. Journal of Research in Science Teaching: The Official Journal of the National Association for Research in Science Teaching 44, 6 (2007), 815-843.

[17] Eun Kyoung Choe, Nicole B Lee, Bongshin Lee, Wanda Pratt, and Julie A Kientz. 2014. Understanding quantified-selfers' practices in collecting and exploring personal data. In Proceedings of the SIGCHI Conference on Human Factors in Computing Systems. 1143-1152.

[18] PhD Christine Seifert. 2019. Do Less: Anti-Productivity Is the New Productivity. (2019). https://bit.ly/3ly9z8g

[19] Francesco Cirillo. 2009. The pomodoro technique. Lulu. com.

[20] Laura Devendorf, Kristina Andersen, and Aisling Kelliher. 2020. Making Design Memoirs: Understanding and Honoring Difficult Experiences. In Proceedings of the 2020 CHI Conference on Human Factors in Computing Systems. 1-12.

[21] George Halkos and Dimitrios Bousinakis. 2010. The effect of stress and satisfaction on productivity. International Journal of Productivity and Performance Management (2010). 
[22] Max Hirshkowitz, Kaitlyn Whiton, Steven M Albert, Cathy Alessi, Oliviero Bruni, Lydia DonCarlos, Nancy Hazen, John Herman, Eliot S Katz, Leila Kheirandish-Gozal, and others. 2015. National Sleep Foundation's sleep time duration recommendations: methodology and results summary. Sleep health 1, 1 (2015), 40-43.

[23] Hilary Hutchinson, Wendy Mackay, Bo Westerlund, Benjamin B Bederson, Allison Druin, Catherine Plaisant, Michel Beaudouin-Lafon, Stéphane Conversy, Helen Evans, Heiko Hansen, and others. 2003. Technology probes: inspiring design for and with families. In Proceedings of the SIGCHI conference on Human factors in computing systems. 17-24.

[24] Anders Højmose. 2010. Daily Stack. (2010). http://everyoneelse.net/projects/daily-stack/

[25] Nassim Jafarinaimi, Jodi Forlizzi, Amy Hurst, and John Zimmerman. 2005. Breakaway: An Ambient Display Designed to Change Human Behavior. In $\mathrm{CHI}$ '05 Extended Abstracts on Human Factors in Computing Systems (CHI EA '05). Association for Computing Machinery, New York, NY, USA, 1945-1948. DOI : http://dx.doi.org/10.1145/1056808.1057063

[26] Ton Jeurissen and Ivan Nyklíček. 2001. Testing the vitamin model of job stress in Dutch health care workers. Work \& Stress 15, 3 (2001), 254-264.

[27] Anat Keinan and Ran Kivetz. 2008. Productivity mindset and the consumption of collectable experiences. ACR North American Advances (2008).

[28] Young-Ho Kim, Eun Kyoung Choe, Bongshin Lee, and Jinwook Seo. 2019. Understanding personal productivity: How knowledge workers define, evaluate, and reflect on their productivity. In Proceedings of the 2019 CHI Conference on Human Factors in Computing Systems. 1-12.

[29] Young-Ho Kim, Jae Ho Jeon, Eun Kyoung Choe, Bongshin Lee, KwonHyun Kim, and Jinwook Seo. 2016. TimeAware: Leveraging framing effects to enhance personal productivity. In Proceedings of the 2016 CHI Conference on Human Factors in Computing Systems. 272-283.

[30] Michiel Kompier and Cary L Cooper. 1999. Preventing stress, improving productivity: European case studies in the workplace. Psychology Press.

[31] Richard S Lazarus. 1995. Psychological stress in the workplace. Occupational stress: A handbook 1 (1995), 3-14.

[32] Ian Li, Anind Dey, and Jodi Forlizzi. 2010. A stage-based model of personal informatics systems. In Proceedings of the SIGCHI conference on human factors in computing systems. 557-566.

[33] Ian Li, Anind K Dey, and Jodi Forlizzi. 2011. Understanding my data, myself: supporting self-reflection with ubicomp technologies. In
Proceedings of the 13th international conference on Ubiquitous computing. 405-414.

[34] Nancy R Lockwood. 2003. Work/life balance. Challenges and Solutions, SHRM Research, USA (2003).

[35] Andrés Lucero. 2015. Using Affinity Diagrams to Evaluate Interactive Prototypes. In Human-Computer Interaction - INTERACT 2015, Julio Abascal, Simone Barbosa, Mirko Fetter, Tom Gross, Philippe Palanque, and Marco Winckler (Eds.). Springer International Publishing, Cham, 231-248.

[36] Stephen Purpura, Victoria Schwanda, Kaiton Williams, William Stubler, and Phoebe Sengers. 2011. Fit4life: the design of a persuasive technology promoting healthy behavior and ideal weight. In Proceedings of the SIGCHI conference on human factors in computing systems. 423-432.

[37] Varenya Raj. 2020. Nidra. (2020). https://www.varenyaraj . com/project/nidra

[38] Yvonne Rogers. 2006. Moving on from weiser's vision of calm computing: Engaging ubicomp experiences. In International conference on Ubiquitous computing. Springer, 404-421.

[39] Simone Schramm. 2016. Less Quantified Self-More Qualified You//Konzepte zu visueller und haptisch erfahrbarer Kommunikation von Körperdaten. UP 2016 (2016).

[40] Mark Snyder. 1974. Self-monitoring of expressive behavior. Journal of personality and social psychology 30, 4 (1974), 526.

[41] Howard Taylor, George Fieldman, and Yochanan Altman. 2008. E-mail at work: A cause for concern? The implications of the new communication technologies for health, wellbeing and productivity at work. Journal of Organisational Transformation \& Social Change 5, 2 (2008), 159-173.

[42] Mark Weiser and John Seely Brown. 1996. Designing calm technology. PowerGrid Journal 1, 1 (1996), 75-85.

[43] Gary White, Zilu Liang, and Siobhán Clarke. 2019. A Quantified-Self Framework for Exploring and Enhancing Personal Productivity. In 2019 International Conference on Content-Based Multimedia Indexing (CBMI). IEEE, 1-6.

[44] John Zimmerman, Jodi Forlizzi, and Shelley Evenson. 2007. Research through design as a method for interaction design research in HCI. In Proceedings of the SIGCHI conference on Human factors in computing systems. 493-502. 


\section{APPENDIX}

\section{A. INTERVIEW GUIDE FOR STUDY 1}

\section{Interview foundation}

Context: Understanding the experience of productivity to design a physical device for keeping track of personal productivity from a subjective experience. The study focuses on productivity at home, mostly because of the current pandemic situation. Anyone with an interest in self-awareness in productivity can participate in the study, which will provide a wide range of backgrounds and perspectives.

Outcome: Understand how, when and where people experience and perceive productivity. The interview will also help to gain knowledge about the current methods, if any, currently used to track and measure productivity. This information will guide the design of a tool for tracking and visualising productivity.

Location: The interview will be conducted online, where the participant and the interviewer are in their respective homes. Therefore, the setup will hopefully make the participant more comfortable as he will be able to decide his preferred sitting and environment.

Energy: The formulated questions are not very sensitive, yet we would like the participant to feel comfortable telling their experiences. The energy during the interview should be calm and relaxed, and the participant should enjoy the conversation.

Medium: This interview is conducted at the beginning of the design process. A conversation will provide better knowledge and understanding of the experience and perception of productivity.

\section{Questions and rationale}

\section{QUESTIONS}

Tell me about you? Do you study? Do you work?

Which tools or materials do you normally use the most? Computer? Software?

Hardware? Notebooks? Pen and paper?

What is productivity for you?

How do you determine whether you are productive or not?

Do you think your criteria is influenced by external factors? In which way?

Current COVID situation? A different culture?

How do you feel when you are productive?

Where do you feel more productive? Why?

When do you feel more productive? Why?

What helps you to be productive? Why?

Do you have any ritual/pattern/behaviour before starting a productivity session?

Do you reward yourself for being productive? How? productive? Which ones?

How do you feel when you are NOT productive?

What distracts you from being productive? Why?

Have you ever tried to visualise your productivity? How? Why?

From which kind of tasks/ activities would you like to visualise your productivity performance?

\section{RATIONALE}

Understand the context of the interviewee

Understand the context of the interviewee Understand the user's meaning of the experience of productivity

Understand the criteria they use for asserting their productivity

Understand possible factors that alter or influence this criteria?

Understand productivity from the perspective of emotions and feelings.

Uncover the location where the user is most productive and why

Uncover the time of the day when the user is most productive and why

Know if there's anything the user does to be productive

Uncover any preparation or behavior pre productivity

Uncover any behavior the user does after being/feeling productive

Understand specific activities that enhance productivity to the user

Understand solutions for being productive when they are not

Uncover what hinders their productivity

Understand their interest in visualising productivity

Uncover preferences in activities to visualize productivity 
QUESTIONS

Does it help you to visualize your productivity? Why?

Do you use any tools that track and measure your productivity? Why?

Is there anything, good or wrong, that you would like to highlight these tools?

\author{
Table 1: Questions and rationale for interviews in Study 1 \\ these tools
}

\section{RATIONALE} the user them

Understand how visualizing personal productivity affects

Understand what tools they currently use, if any, for tracking and measuring productivity and how it helps

Understand the strengths and weaknesses of the use of 


\section{B. INTERVIEW GUIDE FOR STUDY 2}

This is a semi-structured interview; therefore, we change some questions based on the participant's response. The questions we want to ask will be like the following questions:

- How was your experience using RU?

- How did RU affect your perception on self-care activities? And your productivity?

- How often did you interact with RU?

- What criteria did you use to determine the importance of an activity? How did you feel when charging RU?

- How did you feel when the hanging weight dropped?

- What did you like and dislike the most?

- Did you have any issues or problems during these days? 


\section{SUMMARY OF THE FINDINGS OF STUDY 1}

\begin{tabular}{|c|c|c|}
\hline \multicolumn{3}{|l|}{ PRODUCTIVITY } \\
\hline Themes & \multicolumn{2}{|c|}{$\begin{array}{l}\text { Goal achievement } \\
\text { Time efficiency } \\
\text { Perception of progress }\end{array}$} \\
\hline Parameters & \multicolumn{2}{|l|}{$\begin{array}{l}\text { Time } \\
\text { Task } \\
\text { Quality }\end{array}$} \\
\hline \multirow{3}{*}{ Influencing factors } & Internal & $\begin{array}{l}\text { Mood } \\
\text { Energy } \\
\text { Sleep }\end{array}$ \\
\hline & External & $\begin{array}{l}\text { Weather } \\
\text { Culture } \\
\text { Mainstream productivity thinking }\end{array}$ \\
\hline & \multicolumn{2}{|c|}{ Motherhood } \\
\hline Feelings & \multicolumn{2}{|c|}{ Success, satisfaction, pride, fulfilment } \\
\hline \multicolumn{3}{|c|}{ UNPRODUCTIVITY } \\
\hline Feelings & \multicolumn{2}{|c|}{ Frustration, uselessness, failure } \\
\hline Acceptance & \multicolumn{2}{|c|}{ Relief, calm } \\
\hline
\end{tabular}




\section{DESIGN PROCESS OF RU}

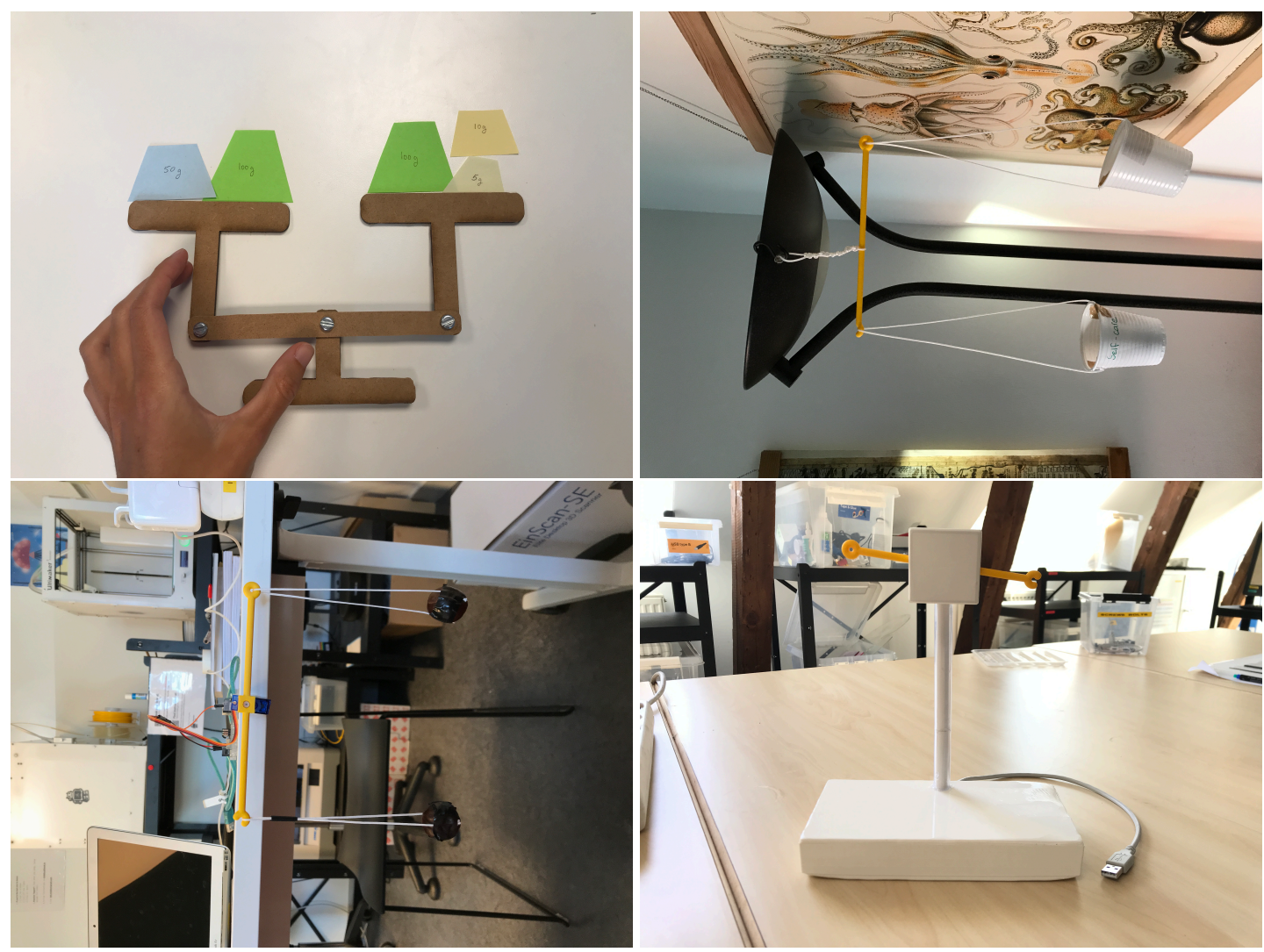

Figure 6. The design process and evolution of prototypes. Top-left: lo-fi prototype made of cardboard for a storyboard. Top-right: prototype used in role playing for a few days at home. Bottom-left: materials and electronics exploration. Bottom-right: early prototype and form of RU 


\section{E. SKETCHING}

These are some of the sketches we did throughtout the design and implementation of RU.

(4)

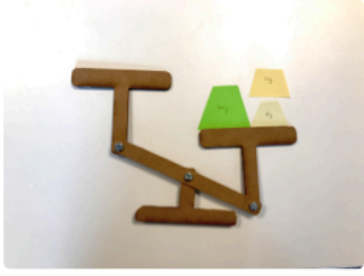

(B)

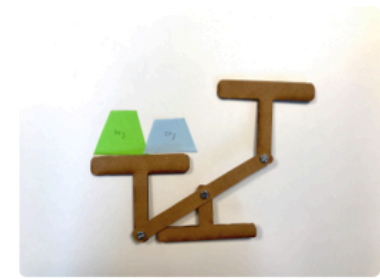

(C)

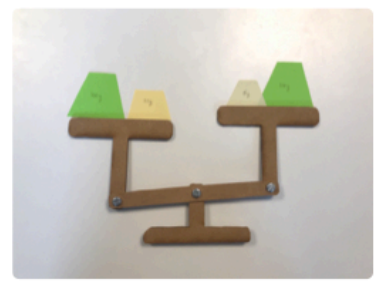

HIGH PRODUCTIVITY

(lead to burnout \& stron)

- Remvind the user

of relf-care

* Add reminder

Repreventation of

Migh SELF-CARE

(foching $\underbrace{}_{\text {change freching }}$

- good gor you

- poritive fecodback (positive reifforcement)

* Balance autorratically when readuing botom.

UNBALANGED BUT FEEING BALALED

- Manual calibration

user curtomization

+ Mr - Reinforcement
- Continuous recording and tracking

- falls when being too producture
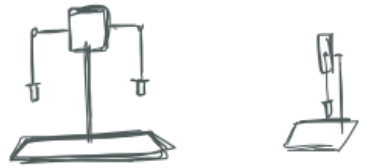

of motor

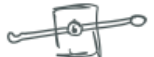

WIFI $\mid$ BLE

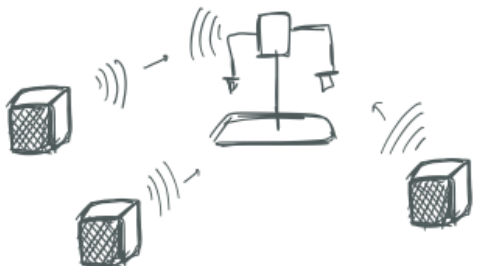

WIfI WI Cloud response

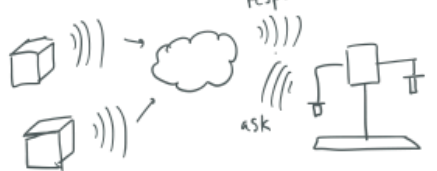

Data is unidirectional 


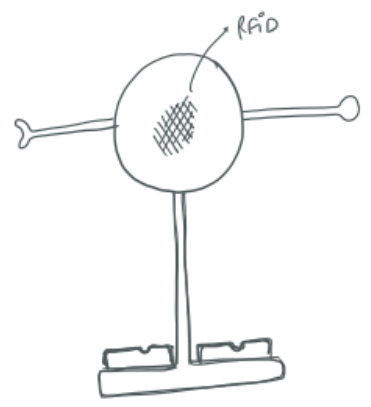

1. Select the card

2- Tap

3- leave it on the pile you want

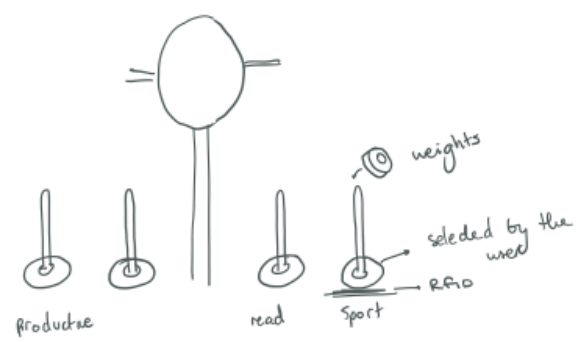

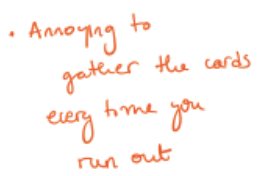

- Tap is not intuitive
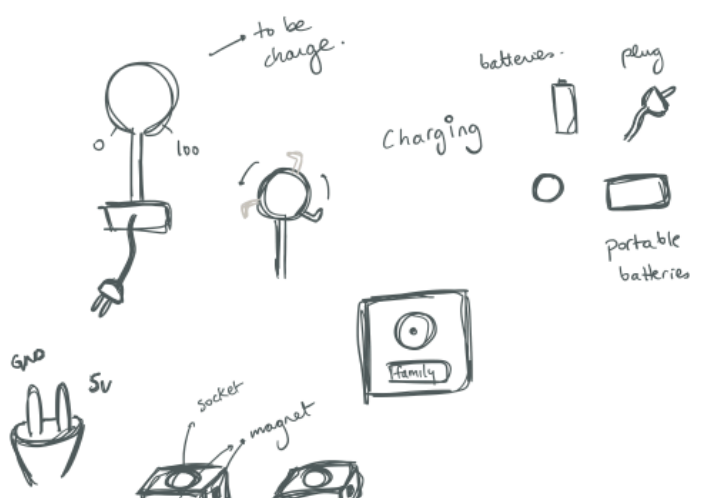

1

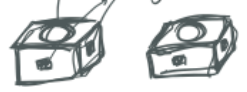

Seting ${ }_{\text {GND }}^{5 \mathrm{NON}}$ 

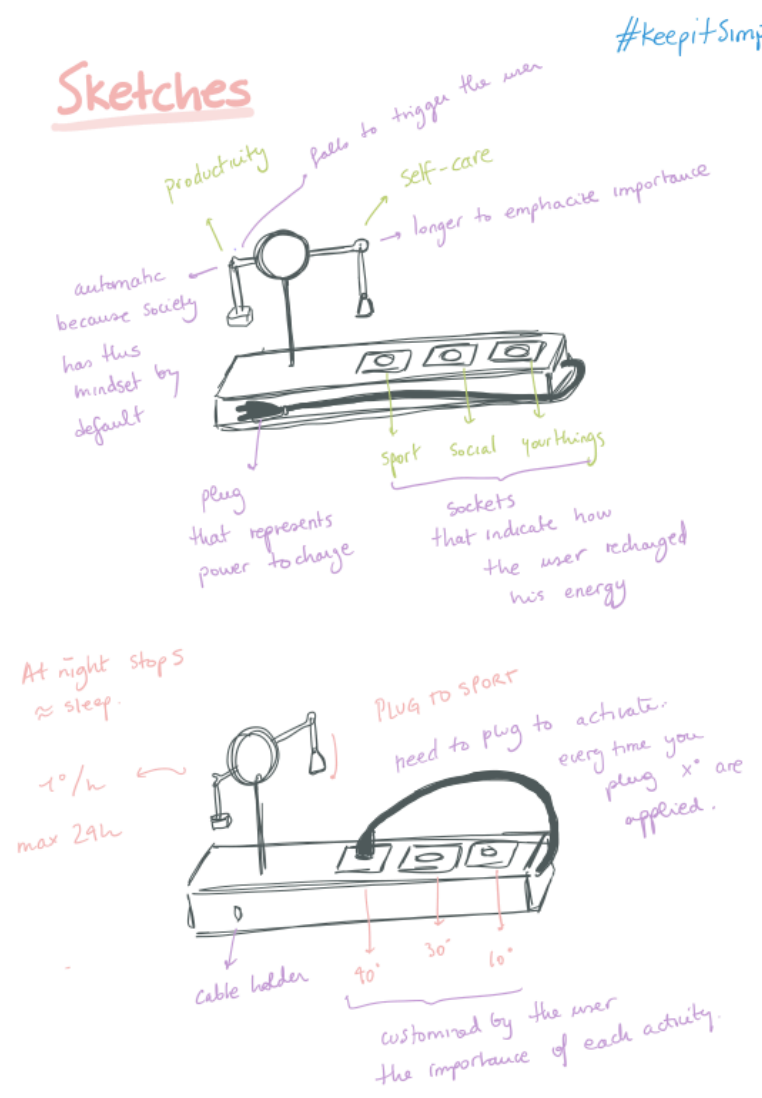


\section{F. DIARY PROBE}

Below, the design of the diary probe we provided to participants in the evaluation study. The booklet was designed in two languages (English and Spanish) and printed in A5 size.
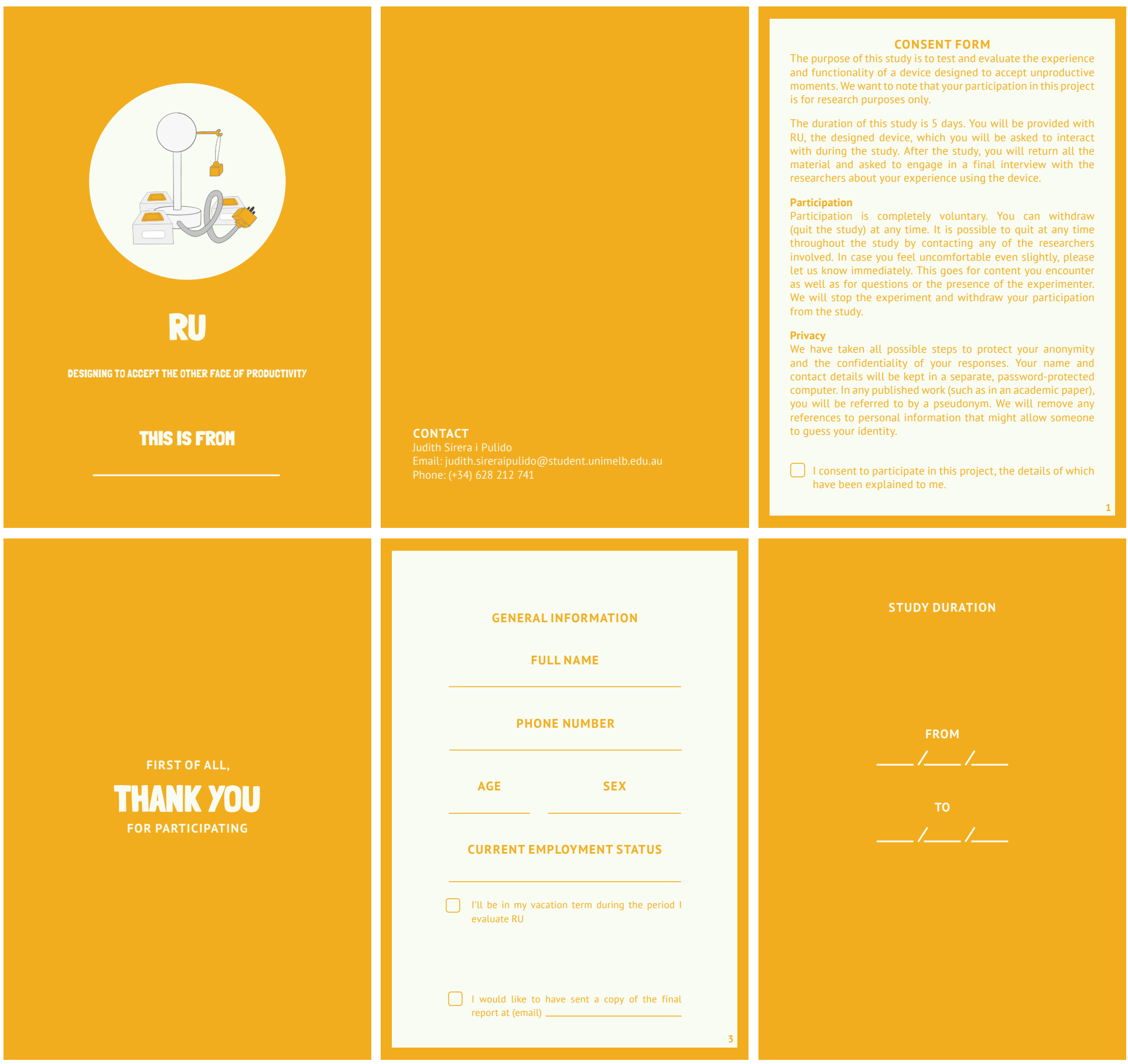


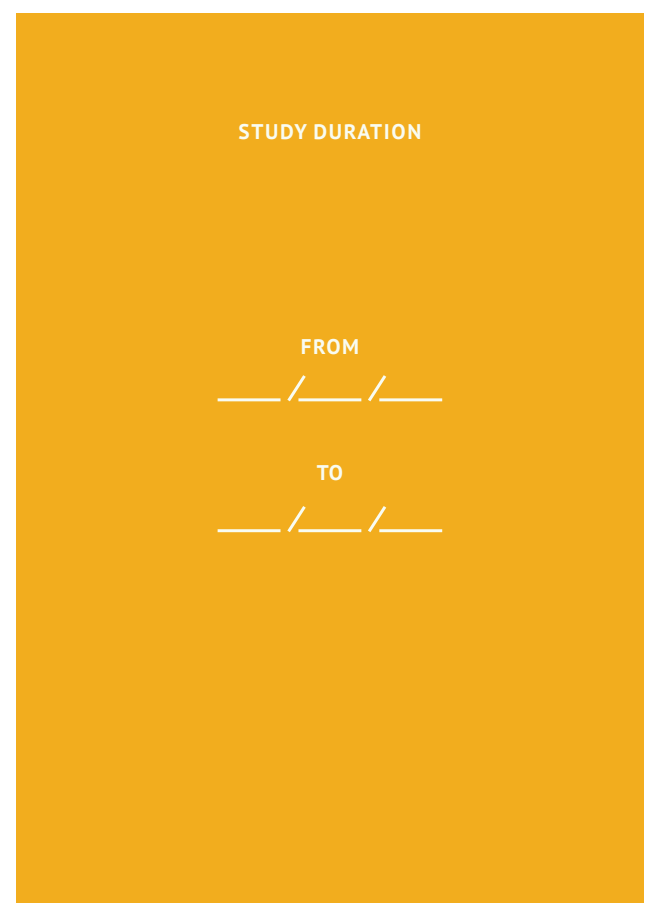

WHERE DID YOU PLACE RU?

Option A

Describe as detailed as possible where did you located RU. Why

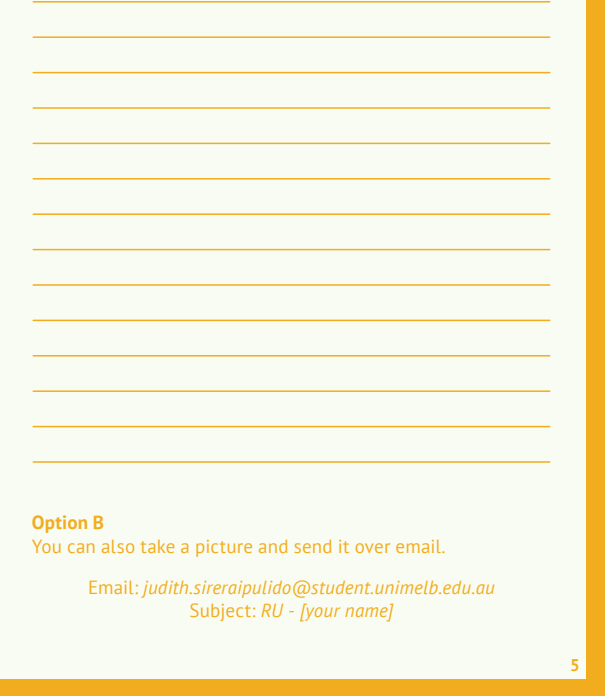

Option B

th.sireraipulido@student.unimelb.edu.au Subject: RU - [your name]

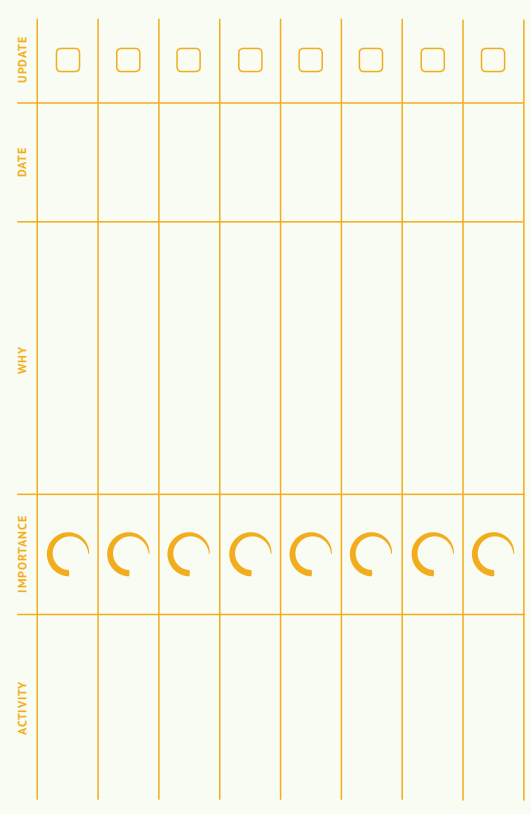
did you place it there?
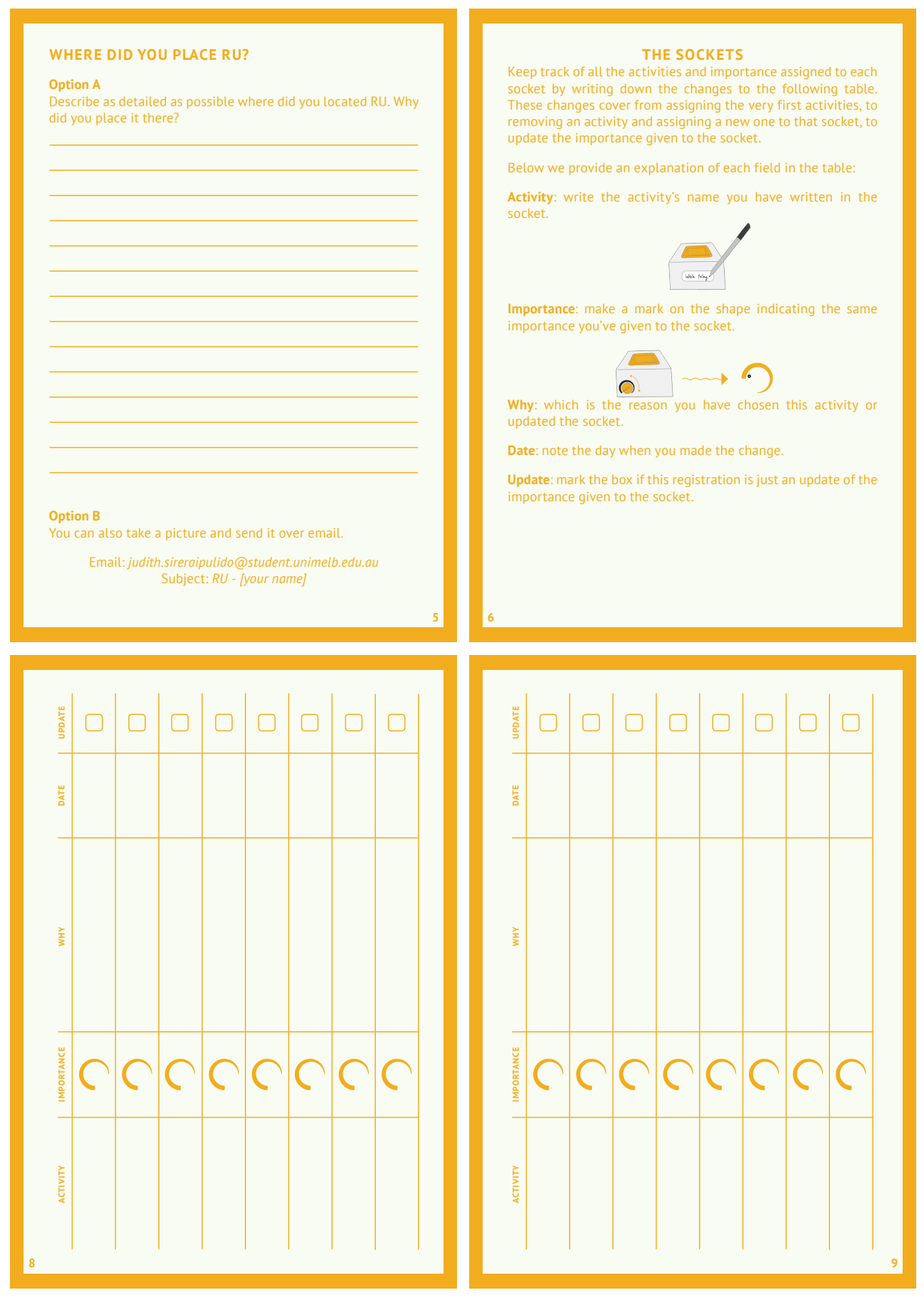

24 

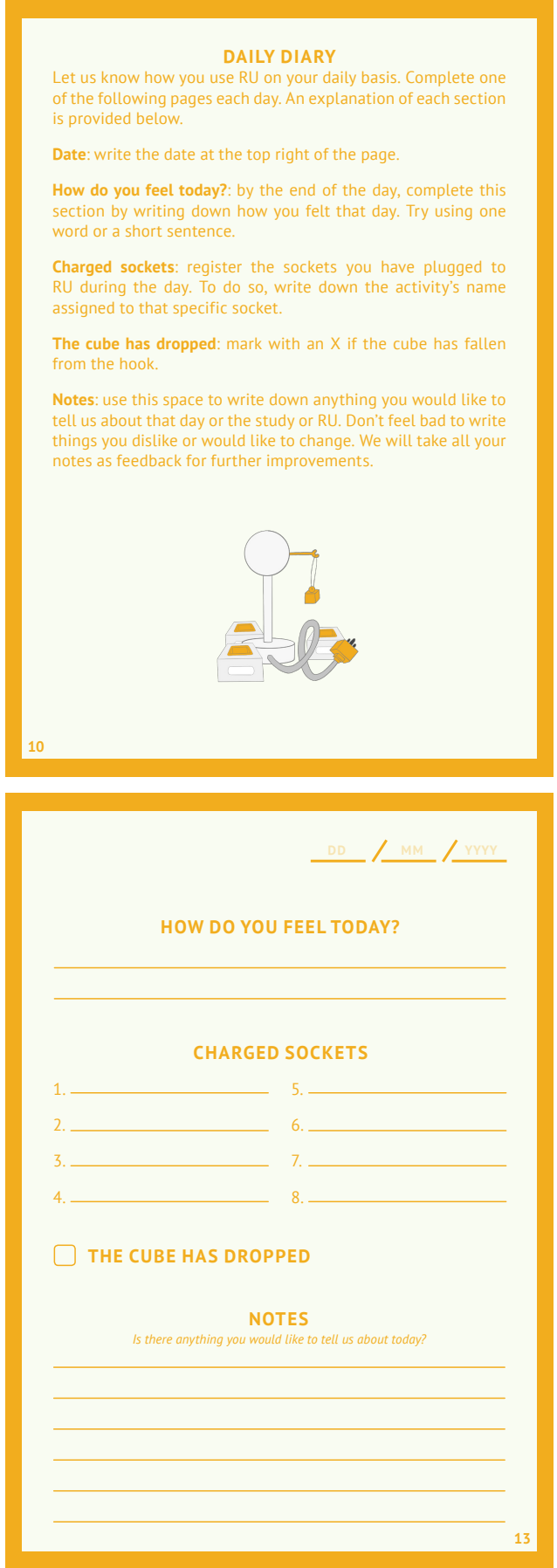
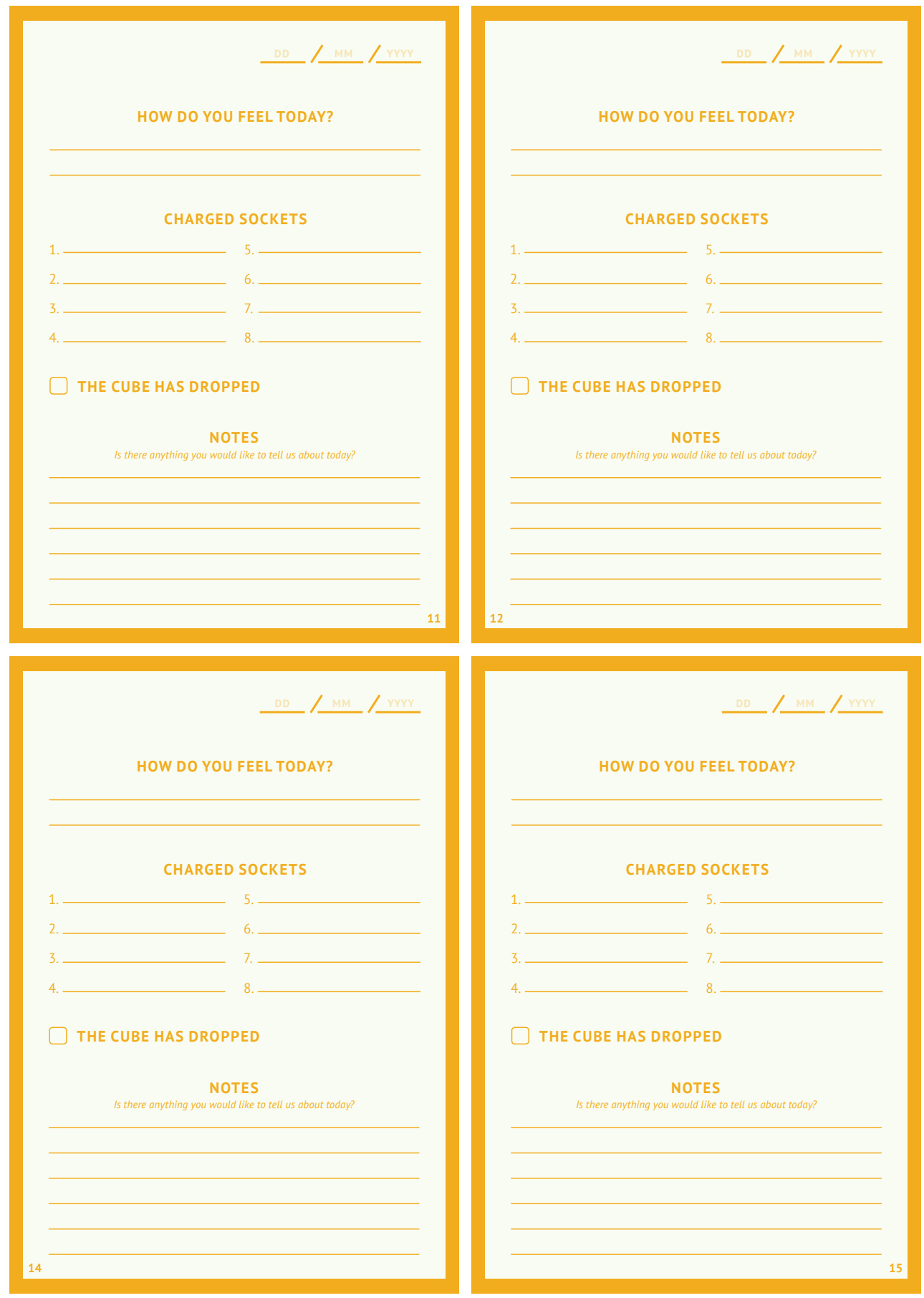

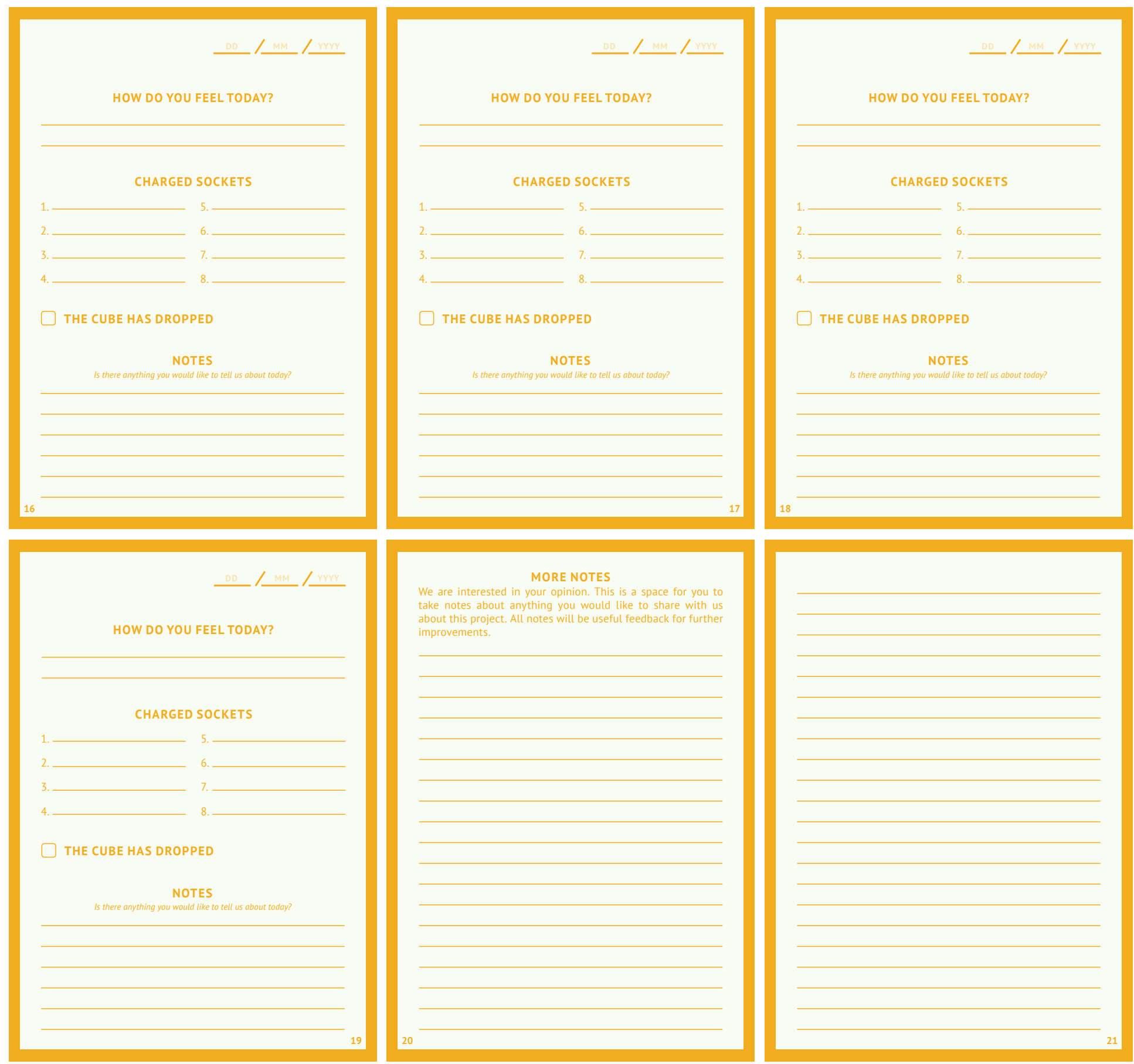


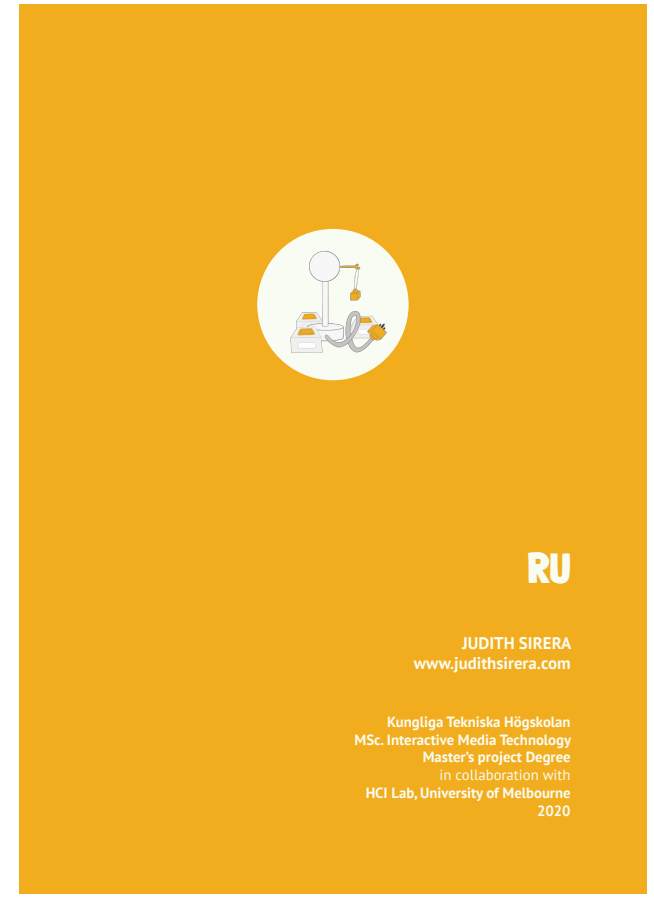




\section{G. INSTRUCTIONS OF USE}

As part of the evaluation kit we provided to participants in the evaluation study, we designed a booklet with the instructions of use. Instructions are described in two languages (English and Spanish). The booklet was printed in A6 size.
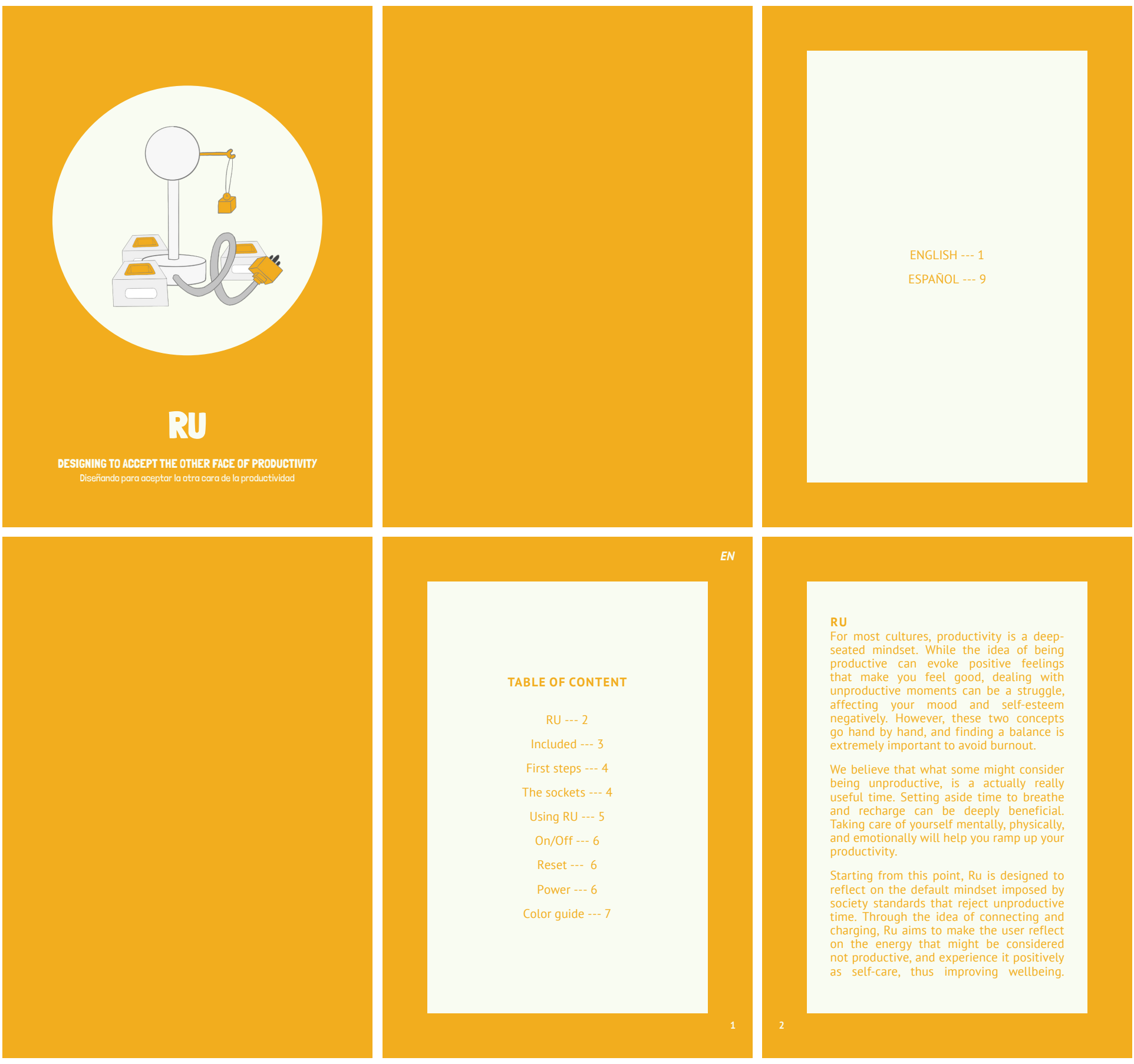

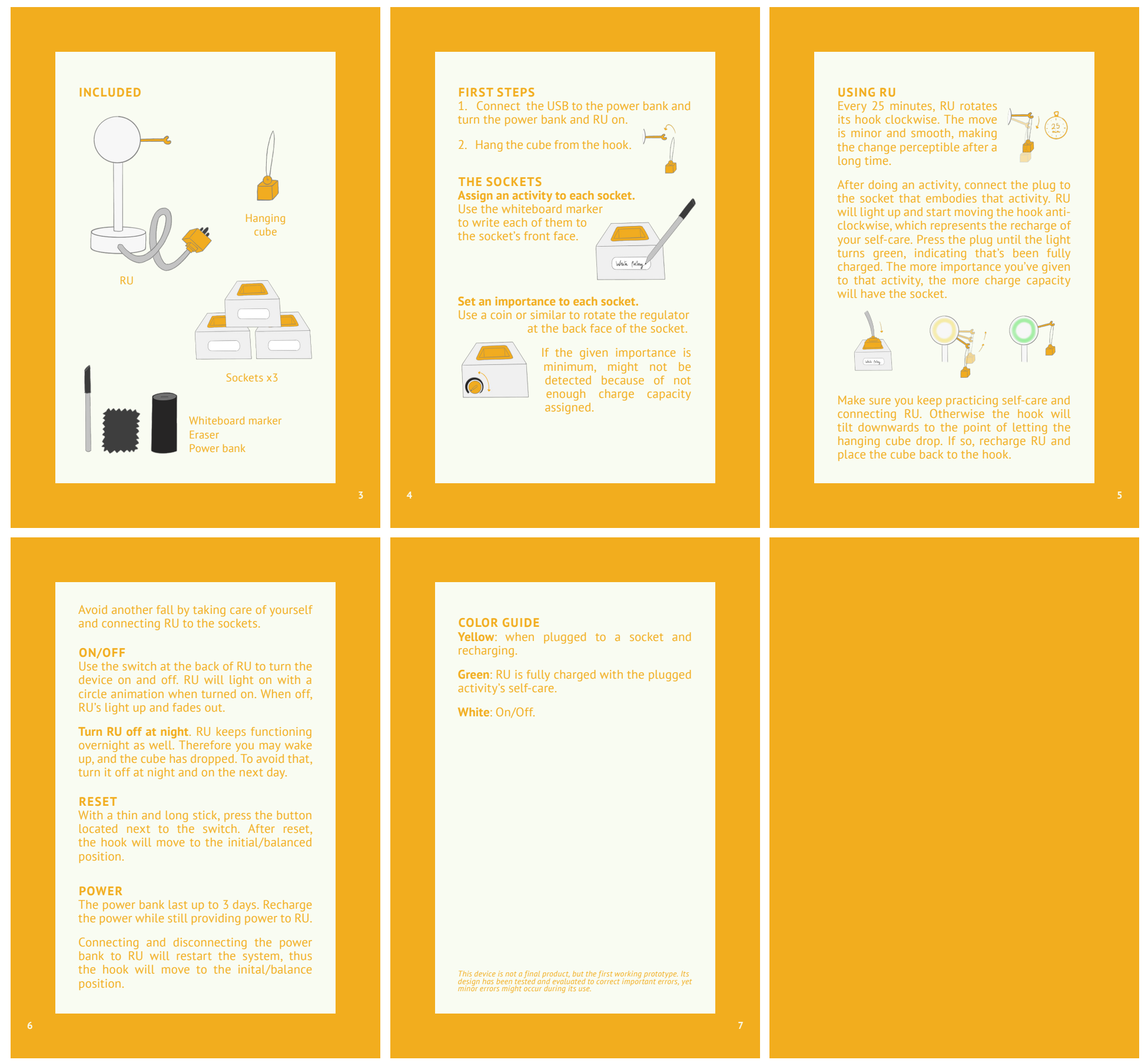

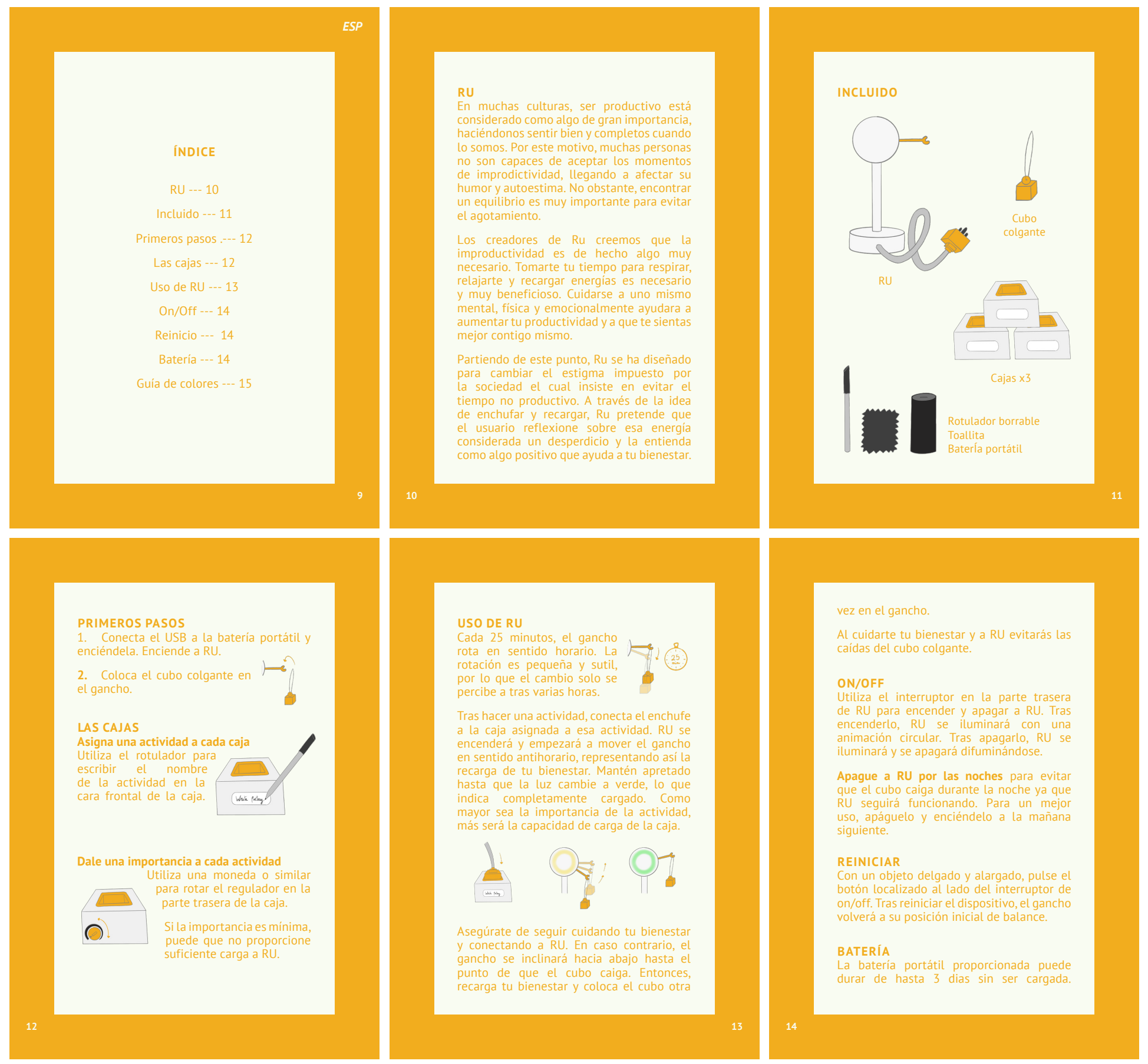

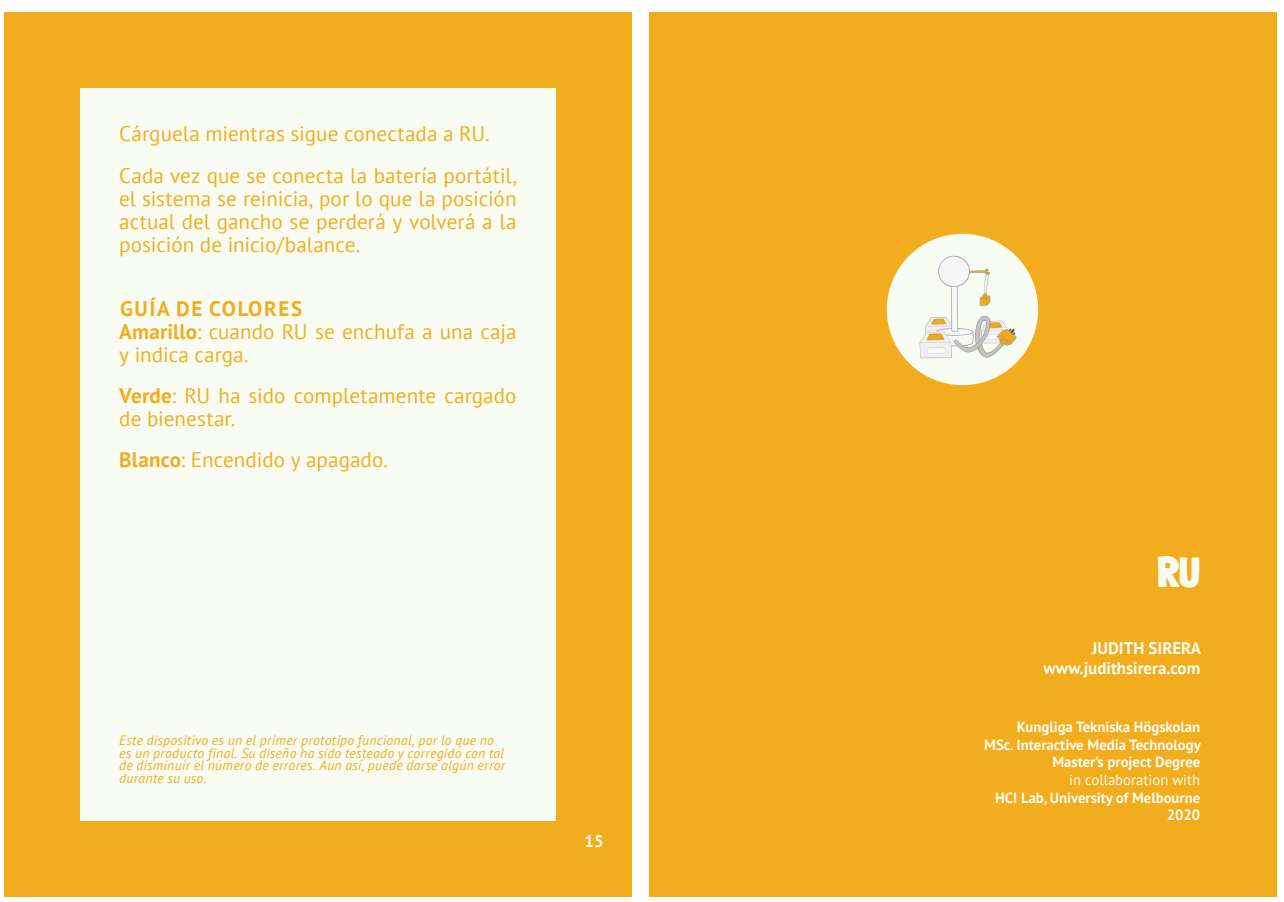
TRITA -EECS-EX-2020:746

www.kth.se 